\title{
MR Fluid Damper and Its Application to Force Sensorless Damping Control System
}

\author{
D. Q. Truong and K. K. Ahn
}

Additional information is available at the end of the chapter

http://dx.doi.org/10.5772/51391

\section{Introduction}

Vibration suppression is considered as a key research field in civil engineering to ensure the safety and comfort of their occupants and users of mechanical structures. To reduce the system vibration, an effective vibration control with isolation is necessary. Vibration control techniques have classically been categorized into two areas, passive and active controls. For a long time, efforts were made to make the suspension system work optimally by optimizing its parameters, but due to the intrinsic limitations of a passive suspension system, improvements were effective only in a certain frequency range. Compared with passive suspensions, active suspensions can improve the performance of the suspension system over a wide range of frequencies. Semi-active suspensions were proposed in the early 1970s [1], and can be nearly as effective as active suspensions. When the control system fails, the semi-active suspension can still work under passive conditions. Compared with active and passive suspension systems, the semi-active suspension system combines the advantages of both active and passive suspensions because it provides better performance when compared with passive suspensions and is economical, safe and does not require either higher-power actuators or a large power supply as active suspensions do [2].

In early semi-active suspension, many researches on variable orifice dampers had been done ([3-4]). With these damper types, regulation on of the damping force can be achieved by adjusting the orifice area in the oil-filled damper, thus changing the resistance to fluid flow, but adjusting the speed is slow because of mechanical motion limitations. Another class of semi-active suspension uses controllable fluids. Two fluids that are viable contenders for development of controllable dampers are: electrorheological (ER) fluids and magnetorheological (MR) fluids. Although the discovery of both ER and MR fluids dates back to the late 1940's, researchers have primarily concentrated on ER fluids for civil engineering applications ([5-8]). Recently developed MR fluids appear to be an attractive alternative to ER fluids for use in controllable fluid dampers [9-15]. 


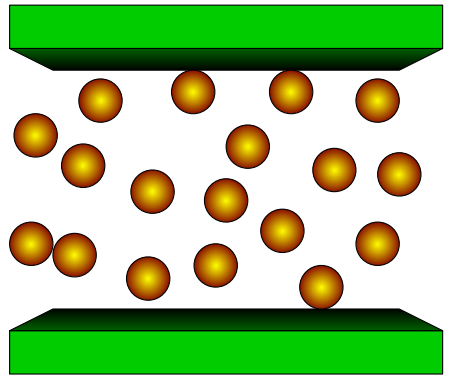

(a) Without Magnetic Field

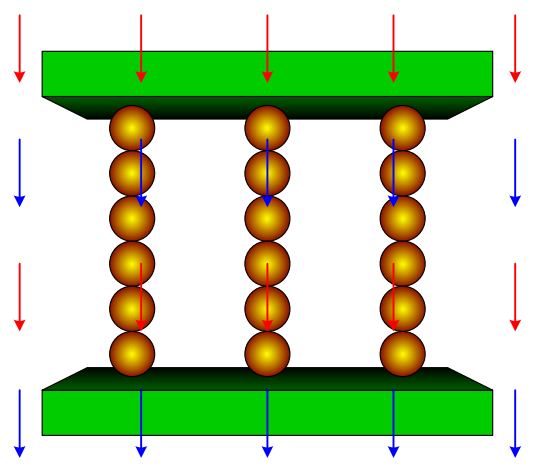

(b)With Magnetic Field

Figure 1. MR fluid - Working principle.

The initial discovery and development of MR fluid can be credited to Jacob Rainbow at the US National Bureau of Standards in the late 1940s [9,10]. These fluids are suspensions of micron-sized, magnetizable particles in an appropriate carrier liquid [11-15]. Normally, MR fluids are free flowing liquids having consistency similar to that of motor oil. However, in the presence of an applied magnetic field, the iron particles acquire a dipole moment aligned with the external field which causes particles to form linear chains parallel to the field, as shown in Fig. 1. This phenomenon can solidify the suspended iron particles and restrict the fluid movement. Consequently, yield strength is developed within the fluid. The degree of change is related to the magnitude of the applied magnetic field, and can occur only in a few milliseconds. A typical MR fluid contains $20-40 \%$ by volume of relatively pure, soft iron particles, e.g., carbonyl iron. These particles are suspended in mineral oil, synthetic oil, water or glycol. A variety of proprietary additives similar to those found in commercial lubricant are commonly added to discourage gravitational settling and promote suspension, enhance lubricity, modify viscosity, and inhibit wear. The ultimate strength of an MR fluid depends on the square of the saturation magnetization of the suspended particles. The key to a strong MR fluid is to choose a particle with a large saturation magnetization. The best available particles are alloys of iron and cobalt that have saturation magnetization of about 2.4 Tesla. Unfortunately, such alloys are prohibitively expensive for most practical applications. The best practical particles are simply pure iron, as they have saturation magnetization of 2.15 Tesla. Virtually all other metals, alloys and oxides have saturation magnetization significantly lower than that iron, resulting in substantially weaker MR fluids. Typically, the diameter of the magnetizable particles is 3 to 5 microns. Functional MR fluids may be made with larger particles; however, particle suspension becomes increasingly more difficult as the size increases. Smaller particles that are easier to suspend could be used, but the manufacture of such particles is difficult. Commercial quantities of relatively inexpensive carbonyl iron are generally limited to sizes greater than 1 or 2 microns.

Due to the special behavior of MR fluid, it has been used for a vast of applications such as: dampers, shock absorbers, rotary brakes, clutches, prosthetic devices, polishing and 
grinding devices, etc. Among them, MR fluid dampers, which utilize the advantages of MR fluids, are semi-active control devices that are widely used in the modern industry nowadays. A typical MR damper includes MR fluid, a pair of wires, a housing, a piston, a magnetic coil, and an accumulator as displayed in Fig. 2a. Here, the MR fluid is housed within the cylinder and flows through a small orifice. The magnetic coil is built in the piston or on the housing. When a current is supplied to the coil, the particles are aligned and the fluid changes from the liquid state to the semi-solid state within milliseconds. Consequently, a controllable damping force is produced. The force procedured by a MR damper depends on magnetic field induced by the current in the damper coil and the piston velocity as in Fig. $2 b$. It is capable of generating a force with magnitude sufficient for rapid response in largescale applications [16-18], while requiring only a battery for power [13]. Additionally, these devices offer highly reliable operations and their performance is relatively insensitive to temperature fluctuations or impurities in the fluid [12]. As a result, there has been active research and development of MR fluid dampers and their applications [9-19,21-29,32,35,36].

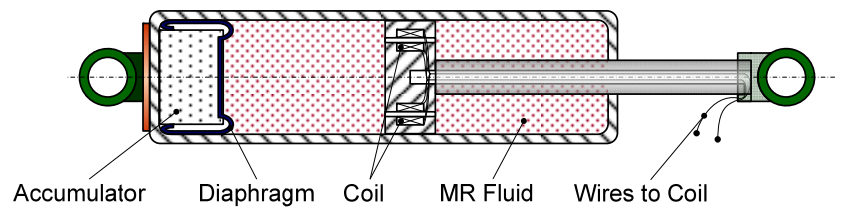

(a) Hardware structure

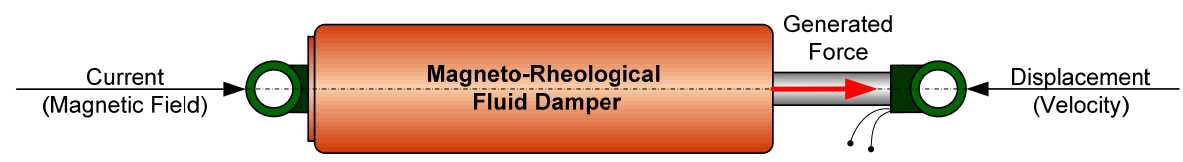

(b) Working principle

Figure 2. General configuration of a MR fluid damper.

However, major drawbacks that hinder MR fluid damper applications are their nonlinear force/displacement and hysteretic force/velocity characteristics. Therefore, one of the challenges involved in creating high efficiencies for MR fluid damper applications, especially in damping control field is to develop an accurate model that can take full advantages of the unique features of this device and to design proper control algorithms in order to improve the system working performances.

With MR fluid dampers modeling technologies, both parametric and non-parametric models have been built by researchers to describe the MR fluid damper behaviors. Savaresi et al [19] made a comparison of both the parametric and non-parametric methods and then developed a complete framework for the development of an accurate model of MRdampers. Parametric models based on mechanical idealizations have been proposed such as Bingham model, Bouc-Wen model, phenomenological model, and others [20-25]. The Bingham model [20] represents the dry-friction as a signum function on the damper velocity and may be considered as a simple model for describing the hysteresis characteristic. The Bouc-Wen model uses a differential equation to depict the non-linear hysteresis with 
moderate complexity and is widely applied in building controls. Once the characteristic parameters of the Bouc-Wen model are determined, the model can obtain the linearity and the smoothness of the transition from the pre-yield to the post-yield region. One of the major problems in the Bouc-Wen model is the accurate determination of its characteristic parameters which is obtained by using optimization or trial error techniques. Consequently, these techniques demand high computational cost to generate the model parameters. Moreover, the fact that each set of constant parameters is valid only for single vibration conditions makes the Bouc-Wen model inappropriate for varying excitation environments. Therefore, many researches on how to develop a MR fluid damper model for higher accuracy and higher adaptability in estimating the behavior of the damper have been done. Spencer et al [21] successfully developed a phenomenological model to improve the model accuracy with an additional internal dynamical variable. Choi and Lee [22] designed a hysteresis damper model based on a polynomial and a curve fitting to predict better the damping force when compared with conventional models. Dominguez et al [23] proposed a methodology to find out the characteristic parameter of Bouc-Wen model and then designed a new non-linear model to simulate the behavior of the MR fluid dampers. Kwok et al designed a hysteretic model based on a particle swarm optimization [24] or using GA technique [25] to modify the Bonc-Wen model and identify the characteristic parameters of the models. The effectiveness of these models with their identification process was proved through the experimental test data. However, the parametric modeling methods require assumptions regarding the structure of the mechanical model that simulates the system's behavior. These approaches could be divergent if the initial assumptions for the model structures are flawed or if the proper constraints are not applied to the parameters [24,25]. Unrealistic parameters such as negative mass or stiffness may be obtained [29].

On the contrary, non-parametric methods could avoid these drawbacks of the parametric approaches for modeling both the linear, nonlinear, and hysteretic systems with high adaptability. For modeling MR fluid dampers, some researches have been done. Chang and Roschke [26] proposed a non-parametric model using multilayer perceptron neural network with optimization method for a satisfactory representation of a damper behavior. Schurter and Roschke [27] investigated the modeling of MR fluid dampers with an adaptive neurofuzzy inference system. The fuzzy structure was simple for modeling; nevertheless, the training model process relied on input and output information on MR fluid dampers and took much computation time. Wang and Liao [28,29] explored the modeling of MR fluid dampers by using a trained direct identification based on recurrent neural network. Although, the designed models could predict the dynamic responses of the dampers with high precision, the model architectures and the training methods were complex.

Once an accurate model for the MR fluid damper is built, it is very useful to investigate the damper characteristics before applying to suspensions. In addition, the well-done model can effectively function as a virtual sensor to estimate the damping force which is used for closed-loop damping control systems with a self-sensing behavior. Self-sensing describes the technique of using a transducer to both actuate and sense concurrently [30,31]. Compared to typical self-sensing damping control systems using separated or integrated actuators and sensors [32], this technique can offer several advantages. A reduction in the 
number of sensing and actuation devices, and associated power, wiring and interfacing, immediately reduces cost and complexity. A sensorless damping control system can also offer higher robustness than the corresponding conventional system in which the failure occurs due to faults in sensor hardware, reading/wiring signal, or measurement noises.

For these reasons and the current demands in MR fluid damper applications, this chapter includes mainly two contents: modeling and damping control technologies. The first one is to revise several typical MR fluid damper modeling methodologies as well as to develop an effective direct modeling method based on a so-called black-box model (BBM) [35,36]. This BBM using a self-tuning fuzzy system based on neural technique is designed to model simply MR fluid dampers and then can be apply to damping control systems as a virtual force sensor. The BBM built in the form of simple fuzzy mapping laws is considered to estimate directly the MR damping force with respect to the MR damper characteristics. In order to improve the accuracy of the suggested model, the back propagation learning rules based on gradient descent method was used to train the fuzzy parameters to minimize the modeling error function. Input information for the model training process is the current supplied for the MR fluid damper and its dynamic responses. The effectiveness of the BBM method as well as the self-sensing ability of a damping system using this model was clearly verified in a comparison with the other methods through modeling and experimental investigations on two damper test rigs. The comparison results show that the BBM has satisfactorily representative ability for the behavior of MR fluid damper with small computational requirement and it can be successfully used as the virtual force sensor for damping systems. The second content is to present a novel damping control methodology which is called force-sensorless damping control. This control technique is based on the developed BBM, and its inverse back-box model, IBBM, which were designed for any given MR fluid damper, to apply to general systems using this damper for damping control with force self-sensing behavior. The IBBM was derived from the optimized BBM and suggested for usage as an effective force controller. In addition, the IBBM structure is online adjusted with respect to the control error to improve the system performance. Consequently, the closed-loop force controller, based on the 'virtual' force sensor - BBM and the adaptive force controller - IBBM, was built for the force-sensorless damping system. Simulations and real-time experiments have been finally carried out to verify that the designed models have satisfactorily representative ability for the behavior of MR fluid damper with small computational requirement and they were successfully applied for forcesensorless control of the damping systems.

\section{MR fluid damper analysis}

\subsection{MR fluid damper}

For later discussions on modeling and control of MR fluid dampers, a MR fluid damper of the small size damper series, RD-1005-3, manufactured by LORD Corporation was used. The damper RD-1005-3 can be adapted to a wide variety of applications because of its simple design, quiet operation, and compact shock absorption with low voltage and current demands that allow for damping control. Specifications of this damper are displayed in Table 1. 
The purpose of this research is to investigate the characteristics of the damper RD-1005-3 which are then used to derive the accurate damper models as well as to design the forcesensorless controller for damping systems using this damper. Therefore, a test rig employing the damper RD-1005-3, named as TR01, is needed for following tasks:

- To perform a series of experiments on this rig for a full investigation of the damper working performance.

- To acquire a set of experimental input/output data which is used to analyze, design and optimize the damper models in a comparison with some typical models.

- $\quad$ Based on the optimized BBM model, the inverse model - IBBM is designed for damping control purpose.

The optimized BBM model as well as the proposed force-sensorless control system based on BBM and IBBM models might be applied not only on this system but also on other damping systems using the same dampers. Consequently, the ability of using these models for modeling and damping control needs to be verified. Therefore, a second damping system, which is named TR02, is indispensable for following purposes:

- $\quad$ This system functions as another typical damping system using the damper RD-1005-3 as same as the one used in the first damping system.

- The optimized BBM model is applied to this system for evaluating the effectiveness of the proposed self-sensing methodology.

- The suggested force-sensorless control method is applied to this rig to evaluate the damping force control performance.

\begin{tabular}{|l|l|}
\hline \multicolumn{2}{|c|}{ LORD MR fluid damper - RD-1005-3 Series } \\
\hline Parameter & Value \\
\hline Compressed length $(\mathrm{m})$ & 0.155 \\
\hline Extended length $(\mathrm{m})$ & 0.208 \\
\hline Weight $(\mathrm{kg})$ & 0.800 \\
\hline Magneto-Rheological fluid & MRF-132DG \\
Viscosity (N-s/m) at $40^{\circ} \mathrm{C}$ & $0.092 \pm 0.015$ \\
Density $\left(\mathrm{kg} / \mathrm{m}^{3}\right)$ & $2980-3180$ \\
Solids content by weight, \% & 80.98 \\
\hline Electrical characteristics: & \\
Maximum input current (A) & 2 \\
Input voltage (VDC) & 12 \\
\hline Mechanical characteristics: & \\
Maximum extension force (N) & 4448 \\
Maximum operating temperature (deg) & 71 \\
\hline Response time (s) & $<0.025$ (time to reach $90 \%$ of max level \\
(amplifier \& power supply dependent) & during 0-1A step input) \\
\hline
\end{tabular}

Table 1. Technical data for the MR fluid damper RD-1005-3

Based on the dimensions and characteristics of the damper RD-1005-3 as well as the design purposes, the two testing systems were designed and set up as described below. 


\subsection{Test rig setup}

\subsubsection{Test rig 01 (TR01) to build the BMM and IBBM models}

The schematic diagram of the test rig TR01 for the damper RD-1005-3 is depicted in Fig. 3a. In this system, a hydraulic actuator and a driving controller (VibMaster) manufactured by Park Electronics were employed to drive the damper. The end-effector of the actuator is a hydraulic cylinder with $0.035 \mathrm{~m}$ diameter which is driven by a servo valve. The servo valve with a nominal operational frequency range of $0-50 \mathrm{~Hz}$, made by Moog Inc., was used as the final control target to adjust the actuator motion. A linear variable differential transformer (LVDT) was set up to measure the piston-rod displacement of the MR fluid damper. In addition, a compatible load cell with $5000 \mathrm{~N}$ capacity made by Bongshin Corp. was attached in series with the damper rod to measure the damping force. A PC installed with the VibMaster control program was used to generate system vibrations, while the PC with a

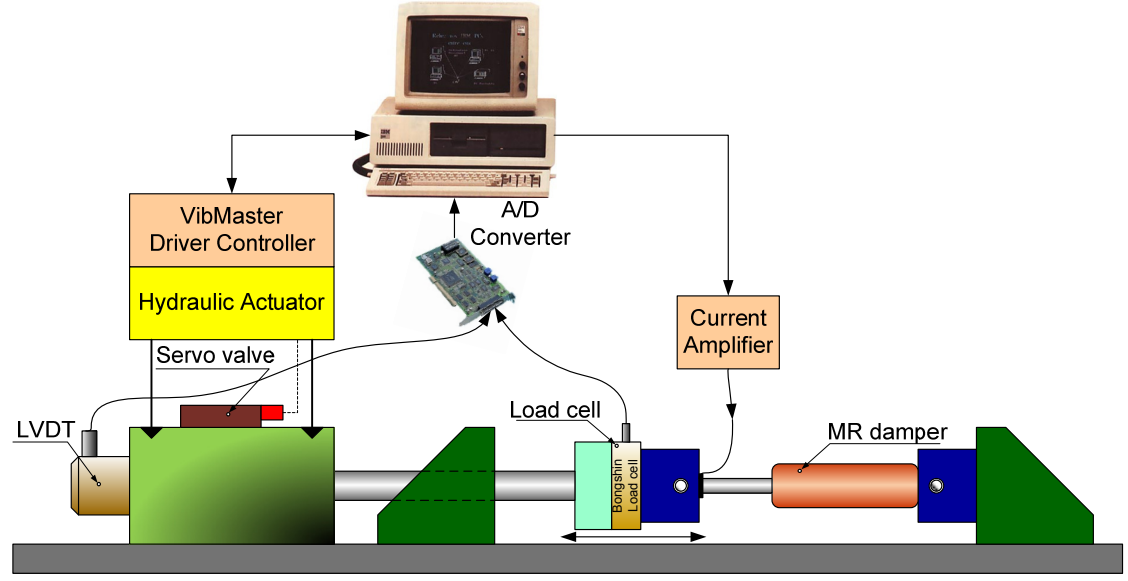

(a) Diagram of TR01

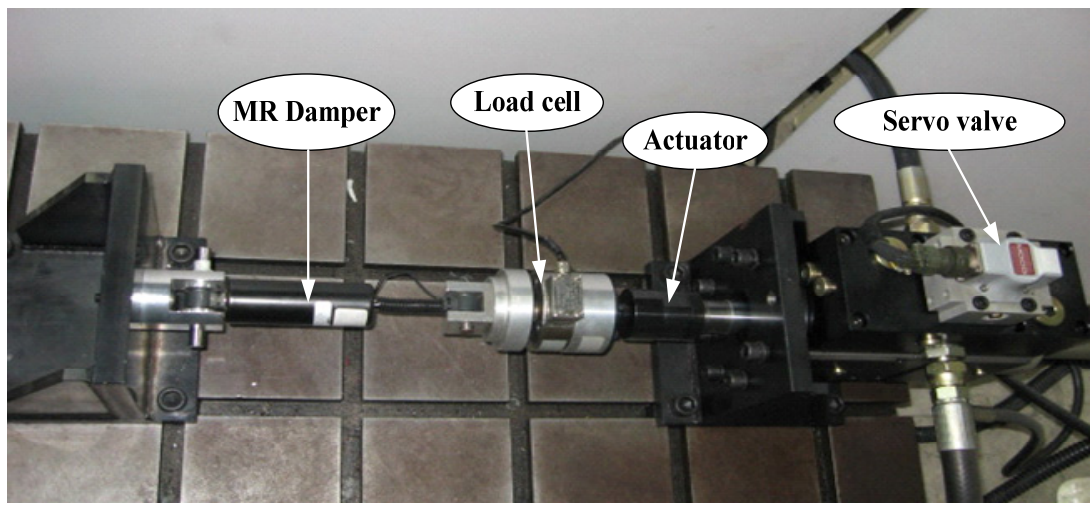

(b) Photograph of TR01

Figure 3. Test rig 01 - TR01 using damper RD-1005-3. 
current amplified circuit sent the current signal to adjust the damper characteristics. Consequently, the feedback signals measured by the LVDT and load cell were sent back to the PC through an Advantech A/D PCI card 1711 to perform a full input-output data acquisition. Finally, the load frame shown in Fig. 3a was designed and fabricated as shown in Fig. $3 \mathrm{~b}$ for the purpose of obtaining the MR fluid damper responses.

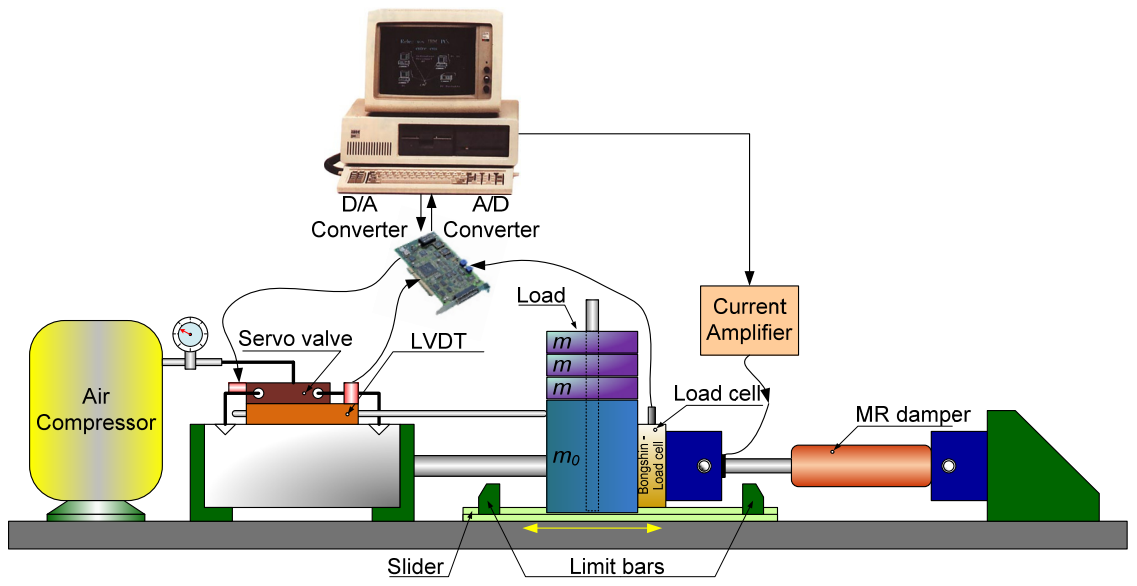

(a) Diagram of TR02

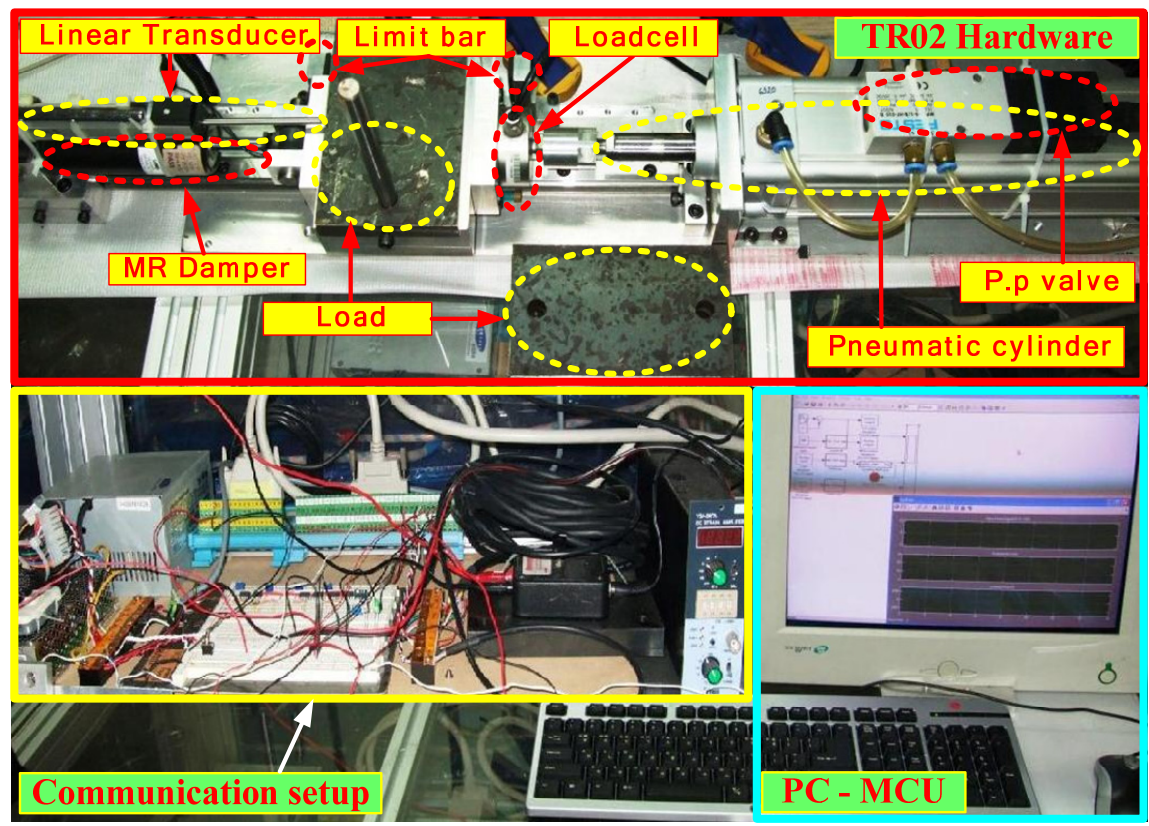

(b) Photograph of TR02

Figure 4. Test rig 02 - TR02 using damper RD-1005-3. 


\subsubsection{Test rig 02 (TR02) to verify the self-sensing behavior and force-sensorless controller}

In order to evaluate the ability of using the proposed self-sensing method and forcesensorless controller for any damping system using the same MR fluid damper, the second test rig (TR02) was set up as depicted in Fig. 4a. The system consists of the MR fluid damper investigated in the TR01 and a pneumatic actuator. The end-effector of this actuator is a pneumatic cylinder with $0.063 \mathrm{~m}$ diameter which is driven by a $5 / 3$-way proportional valve manufactured by Festo Corp. to generate the vibration for the damping system. The spool motion of this servo valve is proportional to its control signal sent from the PC through a D/A converter of the Advantech card 1711. A linear transducer (Novotechnik TR 100) was fixed on the TR02 base and this sensor slider was in contact with the cylinder end-position to feed back the vibration information to the PC. In addition, a loading system was installed in series with the damper rod to create the working environment. Here, the load can be manually varied while the compatible load cell with $5000 \mathrm{~N}$ capacity made by Bongshin Corp. was chosen to measure and feedback the actual damping force in order to compare with the force predicted by the BBM and, consequently, to evaluate the self-sensing method. In addition, the proposed force-sensorless controller is applied to the TR02 to drive the MR fluid damper to follow desired force targets for controller verification.

For safety when doing experiments on the TR02, two limit bars were positioned at two sides of the loading system to restrict the piston movement, consequently, protecting the load cell and MR fluid damper from damages. A photograph of the TR02 configuration is described in Fig. $4 b$.

\subsection{Experiments on the TR01and data analysis}

\subsubsection{Experimental data obtained from the TR01}

To obtain the data used to characterize the RD-1005-3 MR fluid damper behavior, a series of experiments on the rig TR01 was conducted under various sinusoidal displacement excitations while simultaneously altering the magnetic coil in a varying current range. The output of each test was the force generated by the damper. The setting parameters for experiments are listed in Table 2. During all the experiments, the damping force response was measured together with the variation of piston displacement and supplied current for the damper at each step of time, 0.002 second. Fig. 5 depicts an example of relationship between the piston velocity, applied current and dynamic response of the damper corresponding to a sinusoidal excitation with $1 \mathrm{~Hz}$ of frequency and $0.005 \mathrm{~m}$ of amplitude applied to the damper.

\begin{tabular}{|c|c|c|c|}
\hline \multirow{2}{*}{ Test No. } & \multicolumn{2}{|c|}{ Displacement - Sine wave } & \multirow{2}{*}{$\begin{array}{c}\text { MR fluid damper } \\
\text { current }(\mathrm{A})\end{array}$} \\
\cline { 2 - 3 } & Amplitude $(\mathrm{m})$ & Frequency $(\mathrm{Hz})$ & $(0,0.5,0.75,1.0,1.25,1.5)$ \\
\hline 01 to 06 & 0.005 & 1.0 & $(0,0.5,0.75,1.0,1.25,1.5)$ \\
\hline 07 to 12 & 0.005 & 1.5 & $(0,0.5,0.75,1.0,1.25,1.5)$ \\
\hline 13 to 18 & 0.005 & 2.0 & $(0,0.5,0.75,1.0,1.25,1.5)$ \\
\hline 19 to 24 & 0.005 & 2.5 & \\
\hline
\end{tabular}

Table 2. Setting parameters for experiments on the test rig TR01 


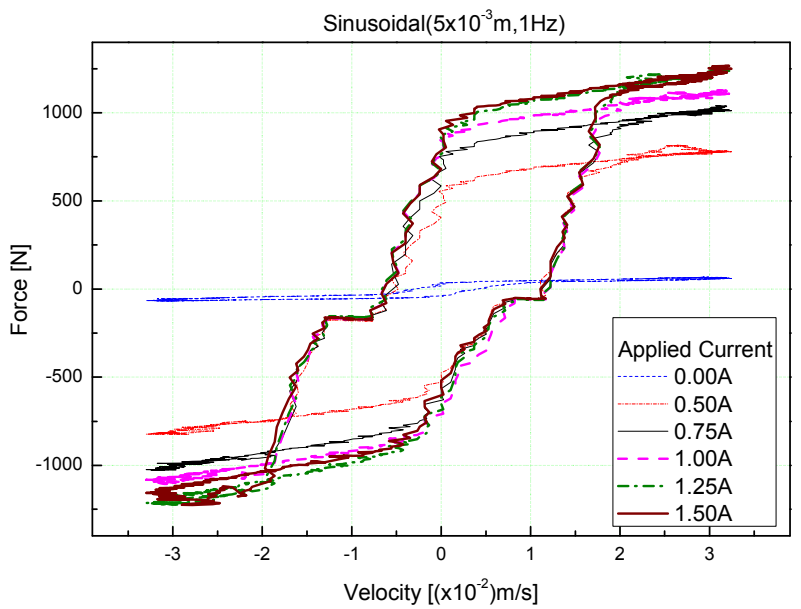

Figure 5. Performance curves for the RD-1005-3 MR fluid damper for a sinusoidal excitation at frequency $1 \mathrm{~Hz}$ and amplitude $0.005 \mathrm{~m}$, and supplied current in range $(0,1.5 \mathrm{~A})$
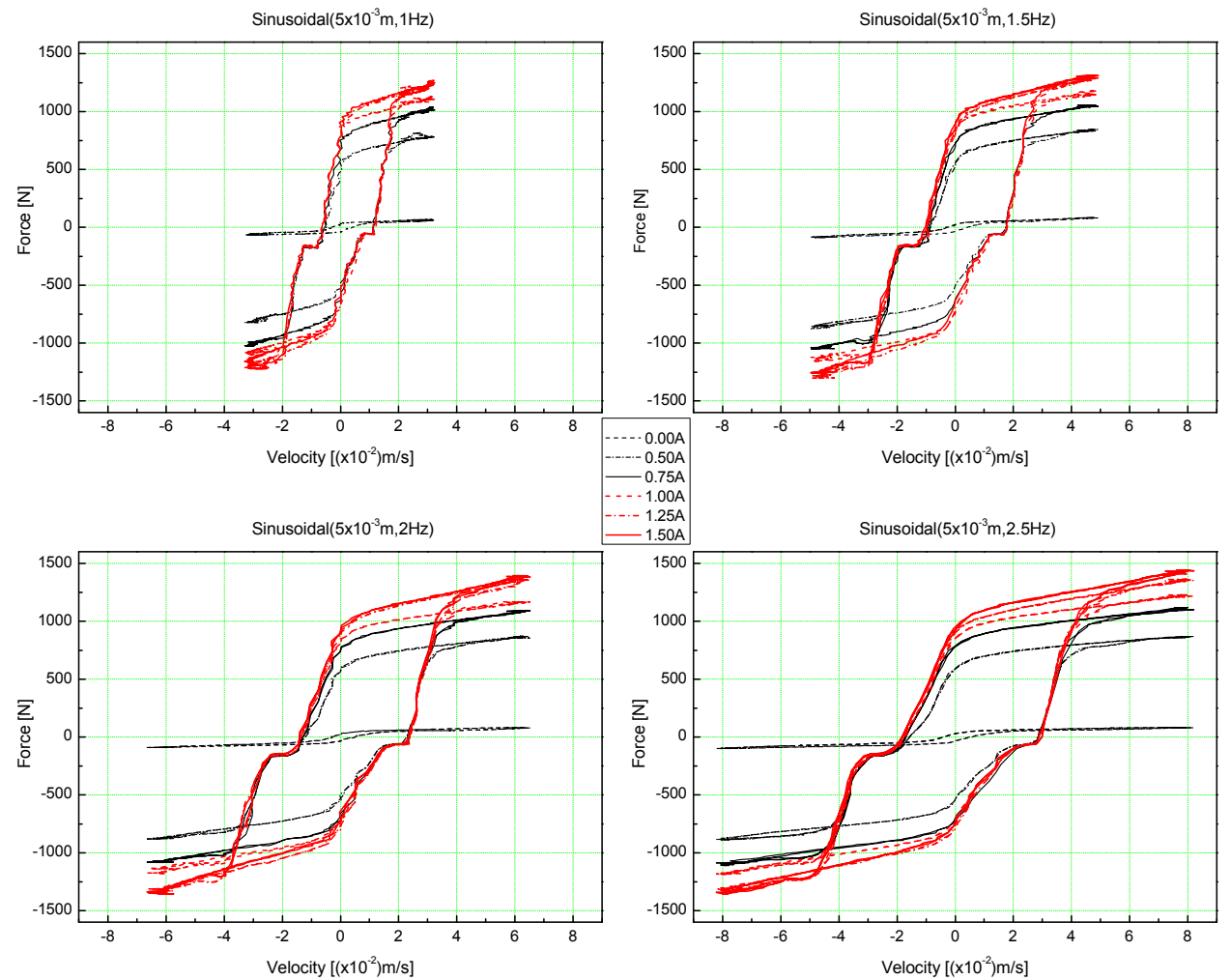

$-1.50 \mathrm{~A}$

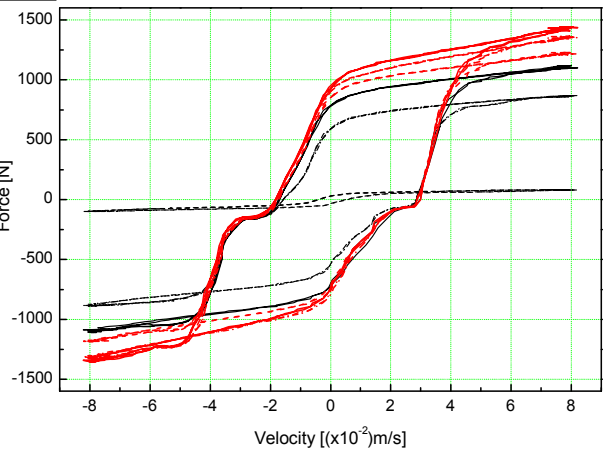

Figure 6. Experimental data measured at sinusoidal excitations (frequency range $(1,2.5) \mathrm{Hz}$, and amplitude $0.005 \mathrm{~m})$, and supplied current in range $(0,1.5) \mathrm{A}$. 


\subsubsection{MR fluid damper characteristic analysis}

Remark 1 (affecting factors). In order to design the MR fluid damper models, an investigation into factors which affect the dynamic responses of the damping system has been done. The first affecting factor is the applied displacement/velocity on the piston rod of the damper. Fig. 6 displays a comparison between damping results under various sine excitations with $0.005 \mathrm{~m}$ amplitude and frequency range from $1 \mathrm{~Hz}$ to $2.5 \mathrm{~Hz}$ while the supplied current level was in range from 0 to $1.5 \mathrm{~A}$. The results show that at fixed current level applied to the damper, the damping force varies due to the piston rod velocity which is caused by the simultaneous change of frequency and/or amplitude of the applied excitation. The second factor affecting the damper behavior is the change in current applied to the damper coil. Fig. 7 shows an example of measurement results in plots of force-time, force-displacement, and force-velocity relations with respect to a $2.5 \mathrm{~Hz}$ sinusoidal excitation and $0.005 \mathrm{~m}$ of amplitude while the current supplied to the damper was in range between 0 and 1.5A. From these figures, it is readily apparent that:

- The force produced by the damper is not centered at zero. This effect is due to the effect of an accumulator containing high pressure nitrogen gas in the damper. The accumulator helps to prevent cavitations in the fluid during normal operation and accounts for the volume of fluid displaced by the piston rod as well as thermal expansion of the fluid.
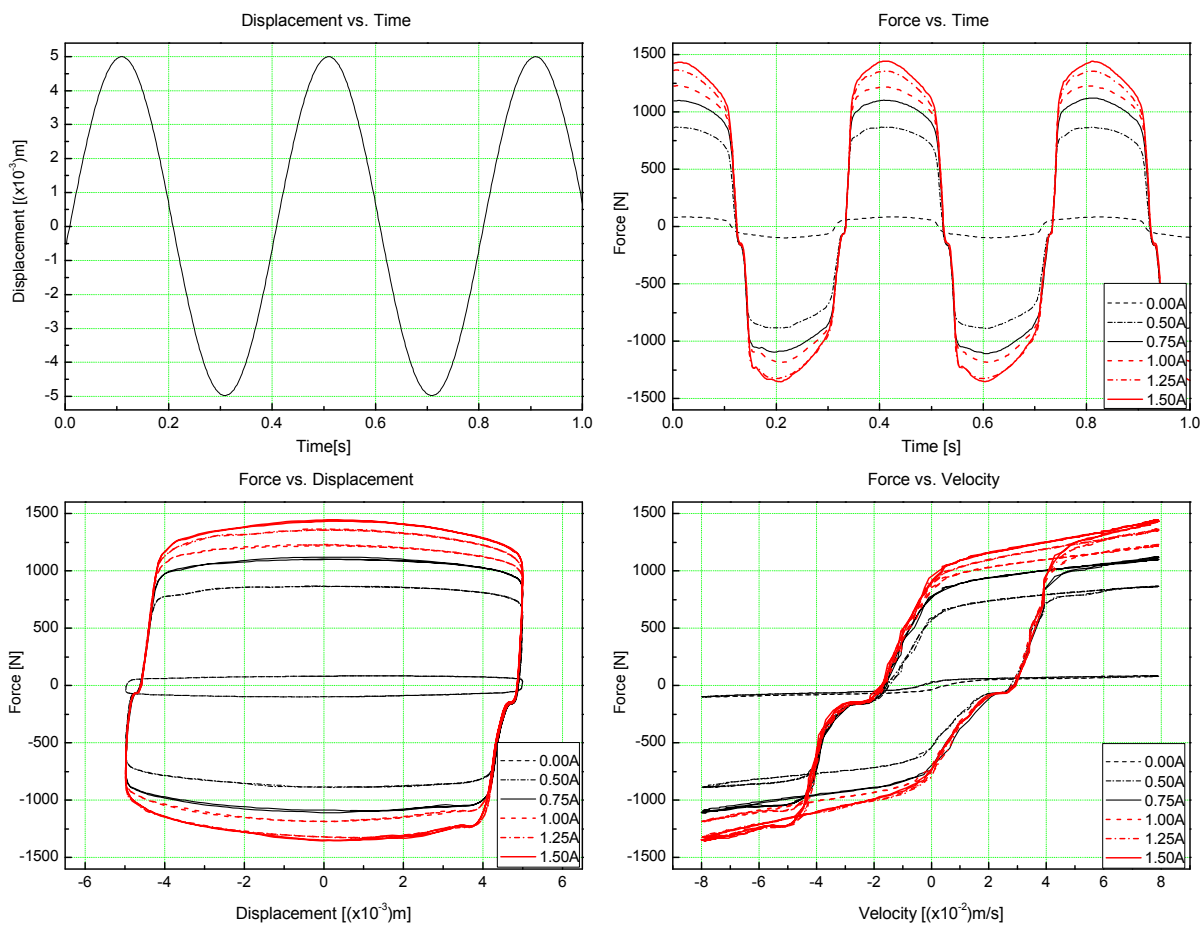

Figure 7. Experimental data measured at sinusoidal excitation (frequency $2.5 \mathrm{~Hz}$, and amplitude $0.005 \mathrm{~m})$, and supplied current in range $(0.5,1.5) \mathrm{A}$. 
- The greater current level, the greater damping force.

- The change rate of force is faster at lower current levels because of the effect of magnetic field saturation.

Based on the above analyses, it is clear that the damping force of the MR fluid damper depends on the displacement/velocity of the damper rod and the current supplied for the coil inside the damper.

\section{MR damper modeling technologies}

\subsection{Typical parametric models}

\subsubsection{Bingham model}

The stress-strain behavior of the Bingham visco-plastic model [37] is often used to describe the behavior of MR fluid. In this model, the plastic viscosity is defined as slop of the measured shear stress versus shear strain rate data. For positive values of the shear rate, $\dot{\gamma}$, the total stress is given:

$$
\tau=\tau_{y(\text { field })}+\eta \dot{\gamma}
$$

where $\tau_{y(\text { field })}$ is the yield stress induced by the magnetic field and $\eta$ is the viscosity of the fluid.

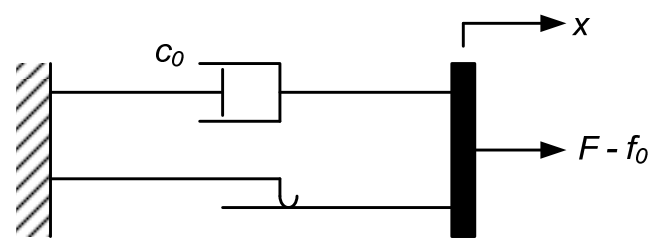

Figure 8. Bingham model of a MR fluid damper.

Based on this model, an idealized mechanical model referred to as the Bingham model was proposed to estimate the behavior of an MR fluid damper by Standway et al [20]. This model consists of a Coulomb friction element placed in parallel with a viscous damper as depicted in Fig. 8. Here, for nonzero piston velocities, $\dot{x}$, the force $F$ generated by the device is given by:

$$
F=f_{c} \operatorname{sign}(\dot{x})+c_{0} \dot{x}+f_{0}
$$

where $c_{0}$ is the damping coefficient; $f_{c}$ is the frictional force related to the fluid yield stress; and an offset in the force $f_{0}$ is included to account for the nonzero mean observed in the measured force due to the presence of the accumulator. Note that if at any point the velocity of the piston is zero, the force generated in the frictional element is equal to the applied force. 

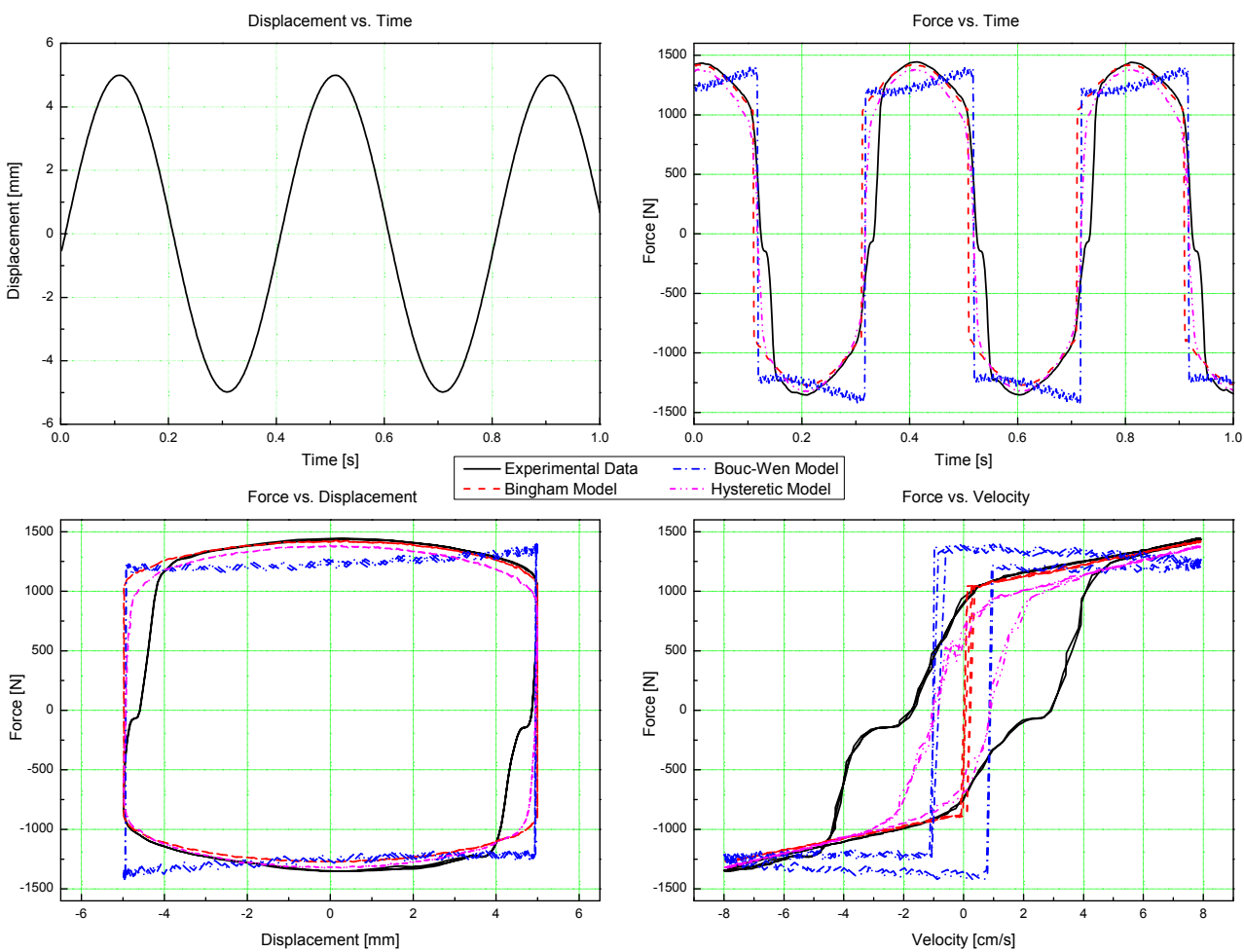

Figure 9. Comparison between experimental data and the predicted damping forces for a $2.5 \mathrm{~Hz}$ sinusoidal excitation with amplitude $5 \mathrm{~mm}$ while current supplied to the damper is $1.5 \mathrm{~A}$.

To present the damper behavior, the characteristic parameters of the Bingham model in equation (2) need to be chosen to fit with the experimental data of the damping system. For example, those parameters were chosen as $c_{0}=50 \mathrm{Ns} / \mathrm{cm} ; f_{c}=950 \mathrm{~N}$ and $f_{0}=75 \mathrm{~N}$ for a $2.5 \mathrm{~Hz}$ sinusoidal excitation with amplitude $5 \mathrm{~mm}$ while the current supplied to the damper was 1.5A. Consequently, the predicted damping force by using the Bingham model was compared with the experimental response as plotted in Fig. 9.

From the results, although the force-time and force-displacement behavior were reasonably modeled, the predicted force-velocity relation was not captured, especially for velocities that were near zero. By using this model, the relationship between the force and velocity was one-to-one, but the experimentally obtained data was not one-to-one. Furthermore, at zero velocity, the measured force had a positive value when the acceleration was negative (for positive displacements), and a negative value when the acceleration was positive (for negative displacements). This behavior must be captured in a mathematical model to adequately characterize the device. Hence, Gamota and Filisko [38] developed an extension of the Bingham model, which is given by the viscoelasticplastic model shown in Fig. 10. 


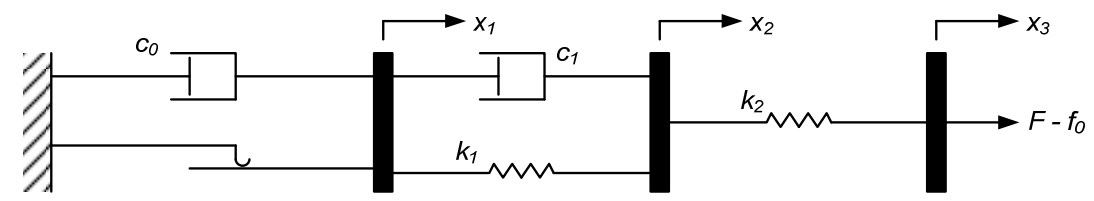

Figure 10. Extened Bingham model of a MR fluid damper.

The model consists of the Bingham model in series with a standard model. The governing equations for this model are given as followings

$$
\left.\begin{array}{rl}
F & =k_{1}\left(x_{2}-x_{1}\right)+c_{1}\left(\dot{x}_{2}-\dot{x}_{1}\right)+f_{0} \\
& =c_{0} \dot{x}_{1}+f_{c} \operatorname{sign}\left(\dot{x}_{1}\right)+f_{0} \\
& =k_{2}\left(x_{3}-x_{2}\right)+f_{0}
\end{array}\right\},|F|>f_{c}
$$

where $c_{0}$ is the damping coefficient associated with the Bingham model; $k_{1}, k_{2}$ and $c_{1}$ are associated with the linear solid material.

This model can present the force-displacement behavior of the damper better the Bingham model. However, the governing equations (3), (4) are extremely stiff, making them difficult to deal with numerically [21]. Therefore, the Bingham model or extended Bingham model are normally employed in case there is a significant need for a simple model.

\subsubsection{Bouc-Wen model}

One model that is numerically tractable and has been extensively used for modeling hysteretic systems is Bouc-Wen model. This model contains components from a viscous damper, a spring and a hysteretic component. The model can be described by the force equation and the associated hysteretic variable as given

$$
\begin{gathered}
F=c \dot{x}+k x+\alpha z+f_{0} \\
\dot{z}=-\gamma|\dot{x}| z|z|^{n-1}-\beta \dot{x}|z|^{n}+\delta \dot{x}
\end{gathered}
$$

where: $F$ is the damping force; $f_{0}$ is the offset force; $c$ is the viscous coefficient; $k$ is the stiffness, $\dot{x}$ and $x$ are the damper velocity and displacement; $\alpha$ is a scaling factor; $z$ is the hysteretic variable; and $\gamma, \beta, \delta, n$ are the model parameters to be identified. Note that when $\alpha$ $=0$, the model represents a conventional damper.

In order to determine the Bouc-Wen characteristic parameters predicting the MR fluid damper hysteretic response, Kwok et al [25] proposed the non-symmetrical Bouc-Wen model with following modifications 


$$
\begin{aligned}
& F=c(\dot{x}-\mu \operatorname{sign}(z))+k x+\alpha z+f_{0} \\
& \dot{z}=\left\{-[\gamma \operatorname{sign}(z \dot{x})+\beta]|z|^{n}+\delta\right\} \dot{x}
\end{aligned}
$$

where $\mu$ is the scale factor for the adjustment of the velocity.

As the optimization results for the test rig applied the damper RD-1005-3 by using GA in [25], the relationships between the Bouc-wen parameters and the supplied magnetization current, $i$, were given as

$$
\begin{aligned}
& c=2.65 \times 10^{3} i+2.05 \times 10^{3} ; k=1.99 \times 10^{3} i+5.57 \times 10^{3} ; \alpha=2.11 \times 10^{3} i+1.68 \times 10^{3} \\
& f_{0}=0.6 i-12.43 ; \mu=-0.02 i+1.25 ; n=0.12 i+1.58 \\
& \delta=0.5 \times 10^{5} i+2.5 \times 10^{5} ; \beta=-0.45 \times 10^{6} i+3.18 \times 10^{6} ; \gamma=0.39 \times 10^{6} i+3.6 \times 10^{6}
\end{aligned}
$$

Because of using the same researched damper, the Bouc-Wen model built from equations from (7) to (9) was tested for modeling the damping force in this study. As a result, the predicted force was plotted as the 'dash-dot' line in Fig. 9 for a $2.5 \mathrm{~Hz}$ sinusoidal excitation with amplitude $5 \mathrm{~mm}$ while the current supplied to the damper was $1.5 \mathrm{~A}$. The estimated damping performance when compared with the real damping performance shows that the proposed Bouc-wen model in [25] could not represent for the damping behavior in the TR01. It is because that the model parameters in equation (9) were only optimized for the damping system using the damper RD-1005-3 in [25]. From the result, it is clearly that to obtain good predicted behavior of a MR fluid damper in a specific system, the Bouc-Wen parameters must be tuned again by using optimization or trial error techniques which causes high computational cost to obtain the optimal parameters.

Furthermore, to obtain better modeling performance, some modified Bouc-Wen models have been proposed. The research results in [21] show that the modified Bouc-Wen model improves the modeling accuracy. However, the model complexity is unavoidably increased with an extended number of model parameters (14 parameters need to be identified in [21]) which may impose difficulties in their identification and take much time for optimization process [27].

\subsubsection{A hysteretic model}

For a simple model, Kwok et at [24] proposed a hysteretic model to predict the damping force of the MR fluid damper RD-1005-3 as illustrated in Fig. 11. The model can be expressed as following equations

$$
\begin{gathered}
F=c \dot{x}+k x+\alpha z+f_{0} \\
z=\tanh (\beta \dot{x}+\delta \operatorname{sign}(x))
\end{gathered}
$$


where: $c$ and $k$ are the viscous and stiffness coefficients; $\alpha$ is the scale factor of the hysteresis; $z$ is the hysteretic variable given by the hyperbolic tangent function; $f_{0}$ is the damper force offset; and $\beta, \delta$ are the model parameters to be identified.

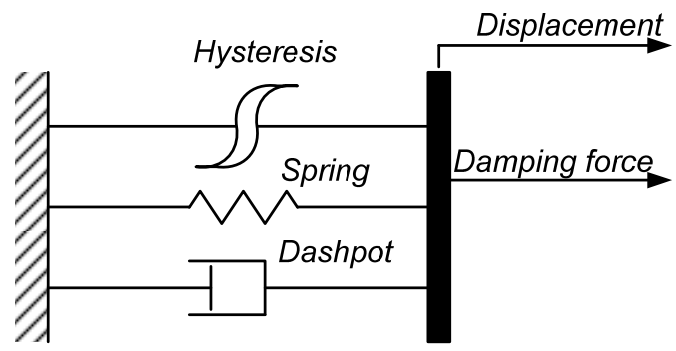

Figure 11. Hysteretic model of a MR fluid damper.

As the results in [24], the parameters in equations (9) and (10) were given:

$$
\begin{aligned}
& \mathcal{c}=1929 i+1232 ; k=-1700 i+5100 ; \alpha=-244 i^{2}+918 i+32 ; f_{0}=-18 i+57 \\
& \beta=100 ; \delta=0.3 i+0.58
\end{aligned}
$$

However, to obtain the parameters as in equation (12), a swam optimization [24] must be used to select the most suitable values with respect to each specific system using the damper RD-1005-3. Hence, when using the set of resulting parameters in [24] to apply to the test system of the MR fluid damper RD-1005-3 in this study, the hysteretic model cannot present well the damper behavior. For example, the modeling result by using the hysteretic model, for a $2.5 \mathrm{~Hz}$ sinusoidal excitation with amplitude $5 \mathrm{~mm}$ while the current supplied to the damper was 1.5A, is depicted in Fig. 9 as the 'short dash' line. The result proves that although the estimated force in this case was better than in case of using Bingham or BoucWen model, the nonlinear characteristic of the damper could not be described well. Moreover, the swam optimization also requires training time to generate the parameters of the hysteretic model.

\subsection{Proposed non-parametric model}

It is known that the typical parametric models show their possibility to be applied for the MR fluid damper identification. However, the decisive parameters of parametric models need to be tuned by using optimization or trial error techniques which causes high computational cost to generate their suitable values. In addition, those models only adapt with specific damping systems. For a new system using the same MR fluid damper series, the optimization process must be done again for a full prediction of the damper behavior [35,36]. Therefore, a non-parametric method based on intelligent techniques, for example, is an effective solution to estimate directly the MR fluid damper behavior with high precision.

Fuzzy system is an intelligent tool imitating the logical thinking of human and then is capable of approximating any continuous function. However, there is no systematic method 
to design and examine the number of rules, input space partitions and membership functions (MFs). Meanwhile, neural network mimics the biological information processing mechanisms. This technique modifies its behavior in response to the environment, and is ideal in case that the expected mapping algorithm is un-known and the tolerance to faulty input information is required. Hence an identification system using fuzzy and neural network theories can be easily selected as an effective method for directly modeling MR fluid dampers purpose.

\subsubsection{BBM model design}

As mentioned in section 2.3, the MR fluid damper force is affected by the rod displacement/velocity and supplied current. Therefore, the designed BBM contains two parts: one is the neural-fuzzy inference (NFI) that is used to estimate the damping force caused by the displacement of the damper rod, and the other is the scheduling gain fuzzy inference (SGFI) which is used to switch between the damping force levels with respect to the current levels supplied for the damper coil. Consequently, the estimated damping force $\left(f_{M R_{-} e s t}\right)$ is computed as a multiplication of the NFI estimated force and the SGFI gain as:

$$
f_{M R_{-} e s t}=K \times U
$$

where: $K$ is the damping force level corresponding to the current level supplied for the damper coil; $U$ is the damping force caused by the displacement applied to the damper rod.

$$
\left\{\begin{array}{l}
K=\alpha_{S G F I} k \\
U=\alpha_{N F I} u
\end{array} ;\left\{\begin{array}{l}
k \in[-1 ; 1] \\
u \in[0 ; 1]
\end{array}\right.\right.
$$

where: $k$ and $\alpha_{S G F I}$ are the SGFI output and a scale factor chosen from the current range for the MR fluid damper coil, respectively; $u$ and $\alpha_{\text {NFI }}$ are the NFI output and a scale factor chosen from the MR fluid damper specifications, respectively.

To evaluate the accuracy of the BBM model, an error function $(E)$ was derived from the difference between the damping force $\left(f_{M R_{-}} e s t\right)$ estimated from the MR model and the actual damping force $\left(f_{M R}\right)$ when the input conditions (current and displacement/velocity) for both the model and real MR fluid damper system are the same. Therefore, the error function is defined as following equation:

$$
E=0.5\left(f_{M R_{-} e s t}-f_{M R}\right)^{2}
$$

Based on the Remark 2, the overall structure of the proposed BBM to model the MR fluid damper is shown in Fig. 12a while the internal structure of the NFI system is described in Fig. 12b. For all of the fuzzy designs, triangle membership functions are used to represent for partitions of fuzzy inputs and outputs. Fuzzy control is applied using local inferences. That means each rule is inferred and the inferring results of individual rules are then aggregated. The most common inference method (aggregation-fuzzy implication operators) 
- max-min method, which offers a computationally nice and expressive setting for constraint propagation, is used. Finally, a defuzzification method is needed to obtain a crisp output from the aggregated fuzzy result. Popular defuzzification methods include maximum matching and centroid defuzzification. The centroid defuzzification is widely used for fuzzy control problems where a crisp output is needed, and maximum matching is often used for pattern matching problems where the output class needs to be known. Hence in this research, the fuzzy reasoning results of outputs are gained by aggregation operation of fuzzy sets of inputs and designed fuzzy rules, where max-min aggregation method and centroid defuzzification method are used.

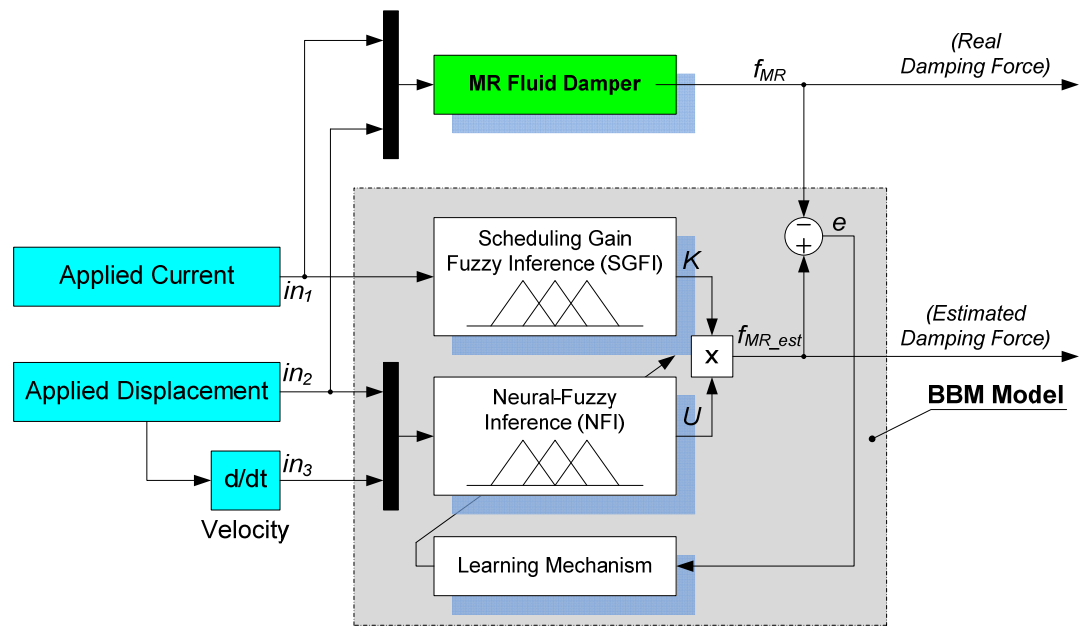

(a) Block diagram for training BBM model

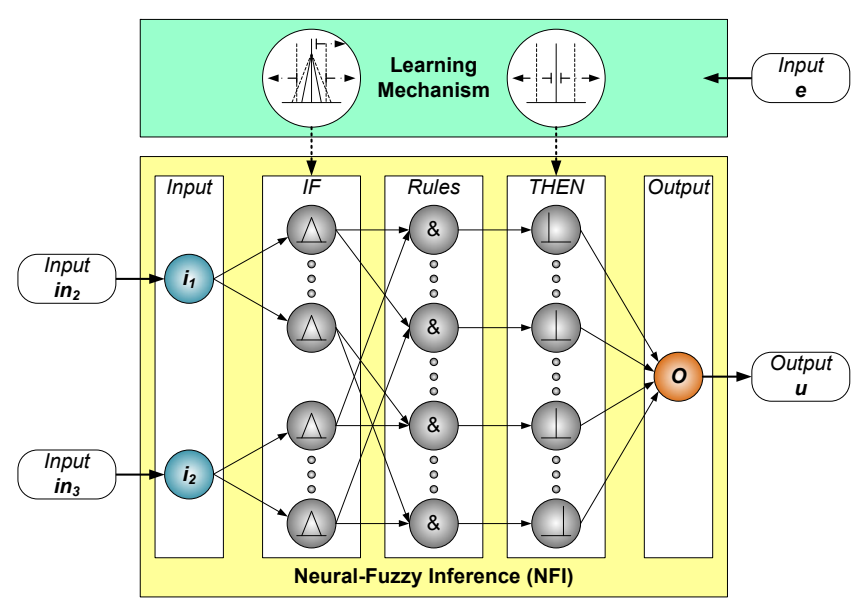

(b) Internal structure of NFI system

Figure 12. Structure of identification for a MR fluid damper using proposed BBM. 


\subsubsection{Neural-Fuzzy inference (NFI)}

The NFI system takes part in estimating the damping force caused by the applied displacement/velocity to the damper. As seen in Fig. 12, the NFI fuzzy set was therefore designed with two inputs ( $i_{2}$, and $\left.i_{3}\right)$ and one output $(u)$. The ranges of these inputs were from -1 to 1 , which were obtained from the applied displacement, and its derivative (velocity) through scale factors chosen from the range of displacement and specifications of the MR fluid damper. The fuzzy output range was also set from -1 to 1 . Consequently, the estimated force can be obtained by multiplying the NFI output and the suitable scale factor $\alpha_{N F I}$ (see equation (2)). The input/output ranges need to be divided into several partitions in order to construct the fuzzy rule map. Based on design experience obtained from the previous researches [33,34], five triangle MFs were used for each the NFI fuzzy input/output for smooth estimating the damping force while it does not require much calculating time consumption.

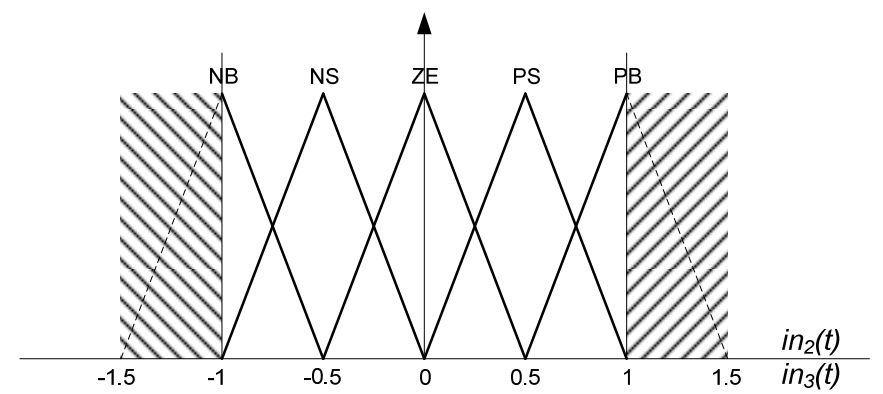

(a) NFI inputs: $\operatorname{in}_{2}(t), \operatorname{in}_{3}(t)$

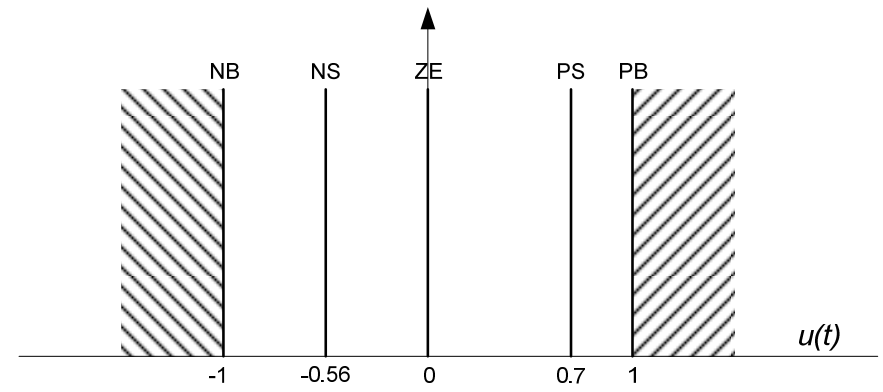

(b) NFI output: $u(t)$

Figure 13. Initial MFs of the NFI inputs and output.

For each input variable, five triangle $\operatorname{MFs}(\mu(.) \in[0,1])$ were designed and named as "NB", "NS", "ZE", "PS" and "PB" which mean "Negative Big", "Negative Small", "Zero", "Positive Small" and "Positive Big", respectively. These MFs and their centroids were initially set with a same shape size and at same intervals, respectively, in Fig. 13a. Because all of the MFs are triangle shapes, so we can express these MFs as follows: 


$$
\mu_{j}\left(x_{i}\right)=\frac{1-2\left|x_{i}-a_{j i}\right|}{b_{j i}}, j=1,2 \ldots \ldots . . . N, i=1 \text { or } 2,\left\{\begin{array}{l}
x_{1} \equiv i n_{2} \\
x_{2} \equiv i n_{3}
\end{array}\right.
$$

where $a_{j}$ and $b_{j}$ are the centre and width of the $j^{\text {th }}$ triangle MF; $N$ is the number of triangles $(N=5)$.

The fuzzy reasoning result of the NFI output is gained using an aggregation operation of fuzzy sets of the inputs and designed fuzzy rules, where the max-min aggregation and centroid defuzzification methods are used. For a pair of inputs (in2, in 3$)$, the NFI output can be computed as:

$$
u=\frac{\sum_{k=1}^{M} \mu\left(w_{k}\right) w_{k}}{\sum_{k=1}^{M} \mu\left(w_{k}\right)}
$$

where: $w_{k}$ and $\mu\left(w_{k}\right)$ are the weight and its height of the NFI output, respectively; $M$ is the number of fuzzy output sets $(M=5)$. The height $\mu\left(w_{k}\right)$ is computed by using the fuzzy output function:

$$
\mu\left(w_{k}\right)=\sum_{i, j} \mu_{i j}\left(w_{k}\right)
$$

where $\mu_{i j}\left(w_{k}\right)$ is defined as the consequent fuzzy output function when the first and second NFI input are in the $i$ and $j$ class, respectively:

$$
\mu_{i j}\left(w_{k}\right)=\delta_{i j} \mu_{i j}
$$

where $\delta_{i j}$ is an activated factor which is active when the input $i n_{2}$ is in class $i$, and the input $i_{3}$ is in class $j ; \mu_{i j}$ is the height of the consequent fuzzy function obtained from the input class $i$ and $j$ :

$$
\mu_{i j}=\min \left[\mu_{i}\left(x_{1}\right), \mu_{j}\left(x_{2}\right)\right] \equiv \min \left[\mu_{i}\left(i n_{2}\right), \mu_{j}\left(i n_{3}\right)\right]
$$

where $\mu_{i}\left(i n_{2}\right)$ and $\mu_{j}\left(i n_{3}\right)$ are obtained from equation (16).

The output $u$ of the NFI system contains five single output values: "NB", "NS", "ZE", "PS", and " $\mathrm{PB}$ ", within the range from -1 to 1 , with the same meaning as the MFs of the inputs. The initial output weights were decided from the experimental results with constant supplied current where the damping force values were caused by the corresponding points of input displacement and velocity [35,36]. Consequently, the output weights were initially set at the different intervals as in Fig. 13b.

By using the above fuzzy sets of input/output variables, experimental data, damper behavior, and experience, the fuzzy rules for the NFI part of the MR model are established 
in Table 3. Five MFs for the each input were used to decide the total twenty five rules by using an IF-THEN structure. Here, one fuzzy rule is composed as follows:

RULE $i$ : IF displacement ( $\left.i n_{2}\right)$ is $A_{i}$ and velocity ( $\left.i n_{3}\right)$ is $B_{i}$ THEN MR force $(u)$ is $C_{i}(i=1,2, . ., 25)$

where $A_{i}, B_{i}$, and $C_{i}$ are the $i^{\text {th }}$ fuzzy sets of the input and output variables used in the fuzzy rules. $A_{i}, B_{i}$, and $C_{i}$ are the linguistic variable values $i n_{2}$, inz, and $u$, respectively.

\begin{tabular}{|c|c|c|c|c|c|c|}
\hline \multirow{2}{*}{$\begin{array}{c}\text { NFI output } \\
\boldsymbol{u}\end{array}$} & \multicolumn{5}{|c|}{ NFI input 02 - Scaled velocity - $\boldsymbol{i n}_{\mathbf{3}}$} \\
\cline { 3 - 7 } & NB & NS & ZE & PS & PB \\
\hline \multirow{3}{*}{$\begin{array}{c}\text { NFI input 01 } \\
\text { Scaled } \\
\text { displacement } \\
\text { in } 2\end{array}$} & NB & NB & NB & NS & ZE & PB \\
\cline { 2 - 7 } & NS & NB & NS & NS & PS & PB \\
\cline { 2 - 7 } & ZE & NB & NS & ZE & PS & PB \\
\cline { 2 - 7 } & PS & NB & NS & PS & PS & PB \\
\cline { 2 - 7 } & PB & NB & ZE & PS & PB & PB \\
\hline
\end{tabular}

Table 3. Rules table for the neural-fuzzy inference of the black box model

As the above description, the fuzzy MFs and rules were dependent on the characteristics of the damper which were investigated from the experimental data. These MFs and rules were then determined from both the intuition and practical experience. There is no systematic method for designing and examining the input space partitions, MFs, and rules which affect directly the modeling quality. As a result, an optimization methodology was indispensible to be used for tuning the NFI structure to fit with the damping behaviors.

Remark 2 (model optimization). In order to improve the identification quality of the proposed models, a learning mechanism using neural network methodology, including the backpropagation algorithm and gradient descent method, has been used to adjust the fuzzy structures of the BBM and IBBM models. The back-propagation algorithm is a first order approximation of the steepest descent technique in the sense that it depends on the gradient of the instantaneous error surface. The algorithm is therefore stochastic in nature which means that it has a tendency to zigzag its way about the true direction to a minimum on the error surface. The basic idea of using the proposed method to optimize the fuzzy designs is to use the back-propagation to tune the input as well as output MF shapes of the models during the system operation process in order to minimize a defined error cost function.

The ability of using the training mechanism based on the back-propagation algorithm and gradient descent method for optimizing the fuzzy scheduling systems were clearly proved in previous researches $[33,34]$. As Remark 2, the proposed optimization method is used to tune the input MF shapes and output weights of the NFI system during the system operation process in order to minimize the modeling error function (15). Consequently, the decisive factors in the inputs MFs $a_{j i}, b_{j i}$, and the output weights $w_{k}$ were automatically adjusted by computing efficiently partial derivatives of the error function $E$ realized by the model network with respect to all these decisive elements. A following set of equations shows the back-propagation algorithm based on the gradient descent method for updating the decisive factors at a step of time $(t+1)^{t h}$ : 


$$
\left.\begin{array}{l}
\left.a_{j i}\right|_{t+1}=\left.a_{j i}\right|_{t}-\left.\eta_{a} \frac{\partial E}{\partial a_{j i}}\right|_{t} \\
\left.b_{j i}\right|_{t+1}=\left.b_{j i}\right|_{t}-\left.\eta_{b} \frac{\partial E}{\partial b_{j i}}\right|_{t} \\
\left.w_{k}\right|_{t+1}=\left.w_{k}\right|_{t}-\left.\eta_{w} \frac{\partial E}{\partial w_{k}}\right|_{t}
\end{array}\right\}
$$

where and are the learning rate which determine the speed of learning; $E$ is the error function defined by (15).

With the self learning of neural network technique and the decreasing of the modeling error, the optimized NFI system works more effectively with high accuracy when compared to the real damping response.

\subsubsection{Scheduling gain fuzzy inference (SGFI)}

This section provides a description of the scheduling gain fuzzy inference which works as an intelligent switch to tune the damping force levels $(k)$ with respect to the current levels supplied for the MR fluid damper. The SGFI system was then designed with a single input (ini) and a single output ( $k$ ) (see Fig. 12).

The range of the input was from 0 to 1 , which was obtained from the supplied current through a scale factor chosen from the current range for the MR fluid damper coil. Five triangle MFs, "Z"(Zero), "VS"(Very Small), “S"(Small), “M"(Medium), and “B”(Big), were used for this input variable. These MFs and their centroids were initially set with a same shape size and at same intervals, respectively, in Fig. 14a. These MFs can be then expressed in the same form as in (16). By using the same fuzzy design method as that of the NFI system in section 3.2.1a to design the SGFI, the output gain $(k)$ corresponding to an input value $(i n 1)$ can be computed as

$$
k=\frac{\sum_{q=1}^{Q} \mu\left(w_{q}\right) w_{q}}{\sum_{q=1}^{Q} \mu\left(w_{q}\right)}
$$

where: $w_{q}$ and $\mu\left(w_{q}\right)$ are the weight and its height of the SGFI output, respectively. $Q$ is the number of fuzzy output sets $(Q=5)$.

For the output $k$ of the SGFI system, five MFs were used. Here, "VS", "S", "M", "B", and "VB" are "Very Small”, "Small”, "Medium", “Big", and "Very Big", respectively. The output range was set from 0 to 1 . The estimated damping force level is then obtained by multiplying the SGFI output and the suitable scale factor $\alpha_{S G F I}$ (see equation (14)). The output weights were decided based on the experimental results and characteristics of the 
MR fluid damper [35,36]. Therefore, the output weights were set as in Fig. 14b. By using the above fuzzy sets of input and output variables, the fuzzy rules for the SGFI part in the BBM model are established in Table 4 by using the IF-THEN structure.

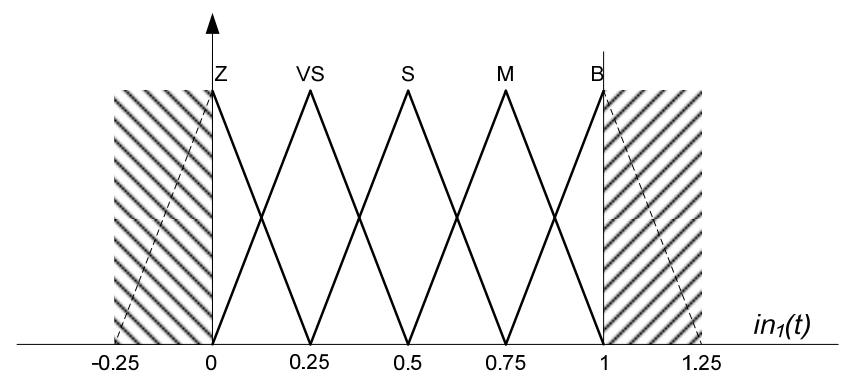

(a) SGFI input: $\operatorname{in}_{1}(t)$

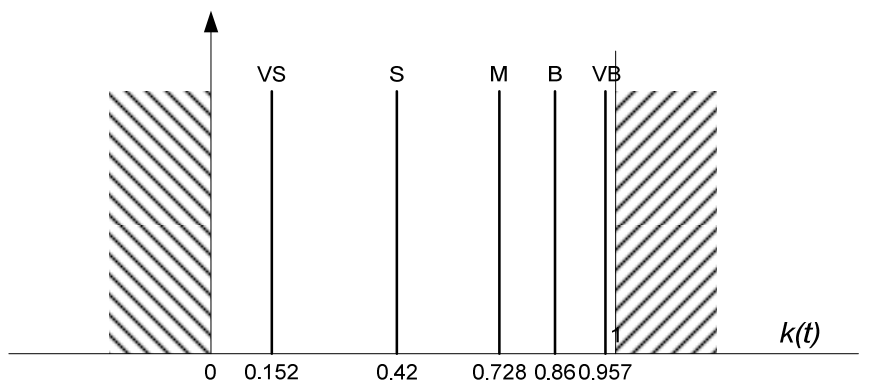

(b) SGFI output: $k(t)$

Figure 14. MFs of the SGFI inputs and output.

\begin{tabular}{|c|c|c|c|c|c|}
\hline $\begin{array}{c}\text { SGFI input - Scaled supplied } \\
\text { current }\left(\text { in }_{1}\right)\end{array}$ & $\mathrm{Z}$ & $\mathrm{VS}$ & $\mathrm{S}$ & $\mathrm{M}$ & $\mathrm{B}$ \\
\hline SGFI output $(k)$ & $\mathrm{VS}$ & $\mathrm{S}$ & $\mathrm{M}$ & $\mathrm{B}$ & $\mathrm{VB}$ \\
\hline
\end{tabular}

Table 4. Rules table for the scheduling gain inference of the black box model

Finally, the output of the proposed black-box model ( $\left.f_{M R_{-} e s t}\right)$ can be computed from the NFI output $(u)$ and SGFI output $(k)$ using equations (13) and (14).

\subsubsection{BBM model verification}

\subsubsection{Comparison between modeling results and experimental data on the TR01}

From $[35,36]$, the model training process and simulations have been carried out to find out the BBM model with optimized parameters and to evaluate the ability of the optimized BBM model when comparing with the measured dynamic responses of the damper, respectively. 
As the result, the BBM with parameters optimized by the leaning mechanism with respect to the modeling error cost function was found. Fig. 15 shows the MFs of the BBM system after training to obtain high accuracy in estimating force of the MR fluid damper. The optimized BBM model was then evaluated in the comparison with the actual measurement data.

Fig. 16 displays the modeling results of the proposed BBM model in a comparison with the real damping behavior for a $2.5 \mathrm{~Hz}$ sinusoidal displacement. The results show that with the designed modeling method, the nonlinear characteristic of the MR fluid damper can be directly estimated with high accuracy for both the force/time, force/displacement, and force/velocity relations despite the variation in applied current for the damper.

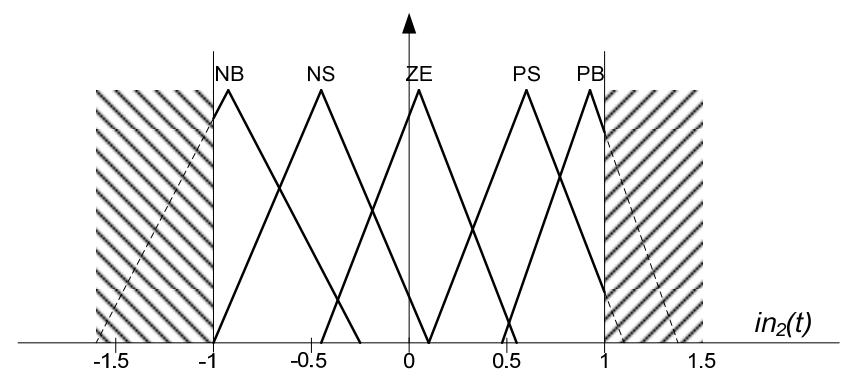

(a) NFI input $i_{2}(t)$ after training

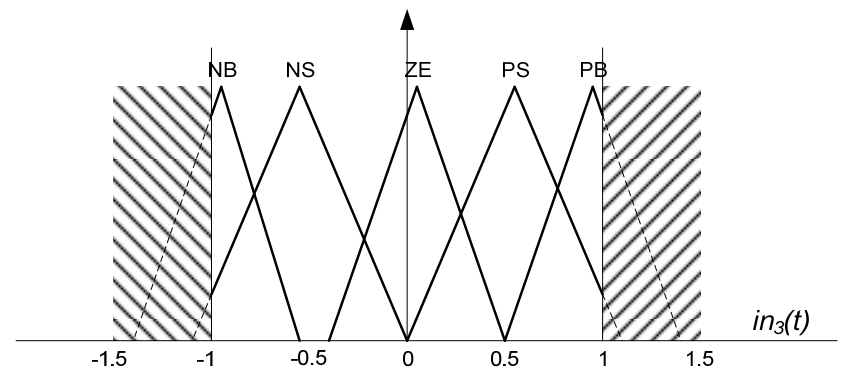

(b) NFI input ins $(t)$ after training

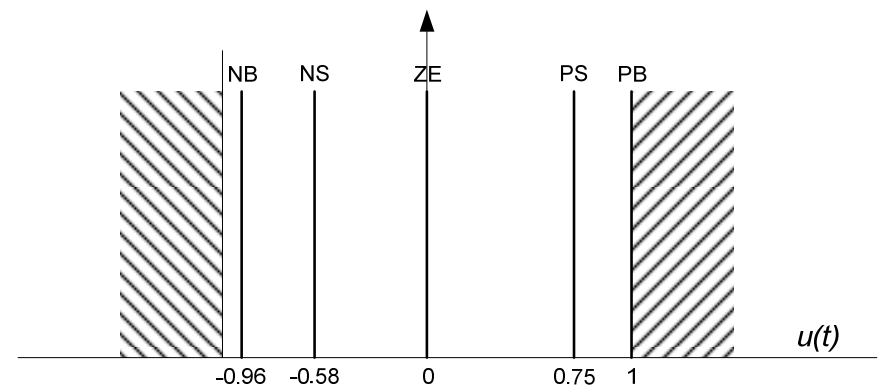

(c) NFI output $u(t)$ after training

Figure 15. MFs of the NFI inputs and output after training. 

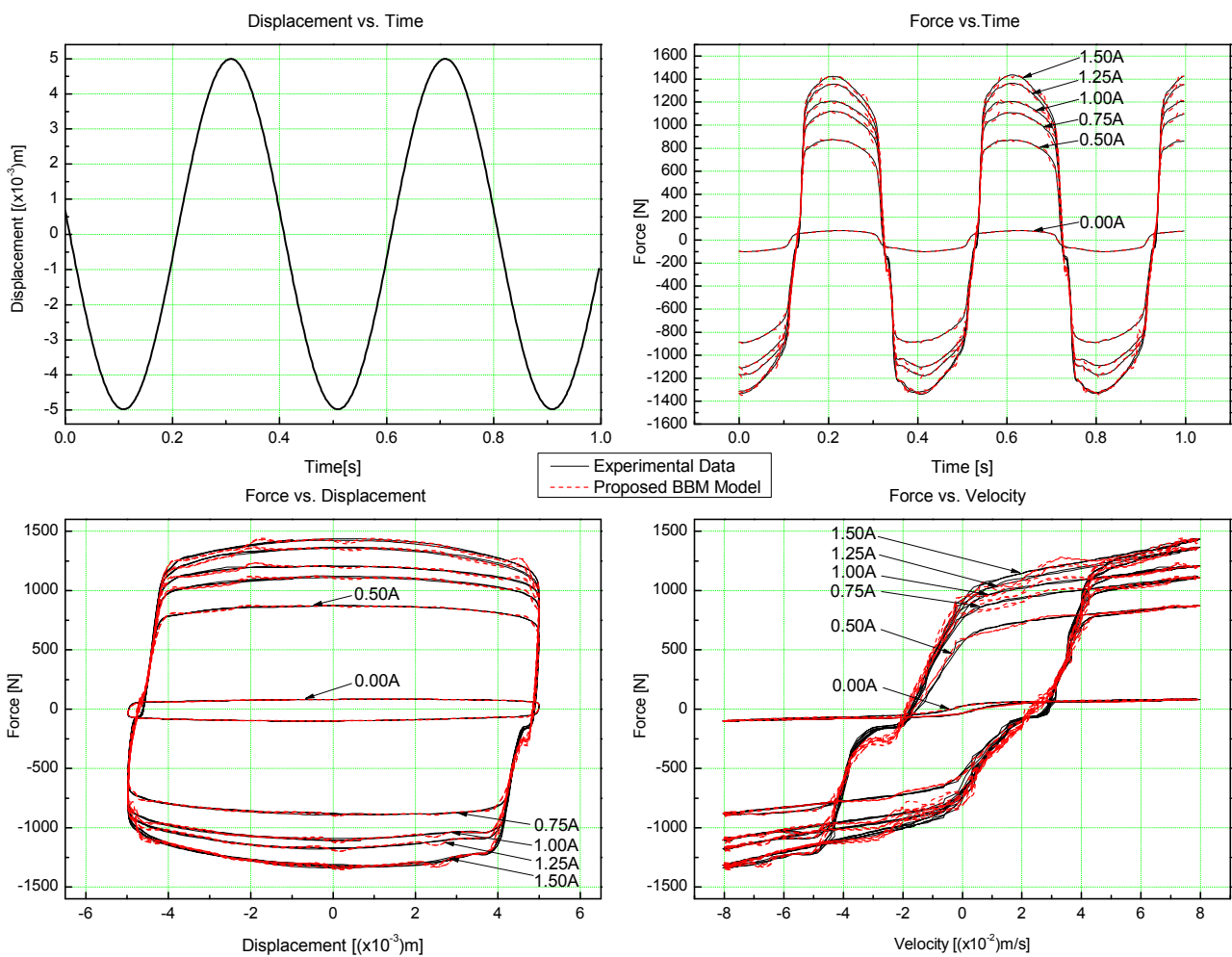

Force vs. Velocity

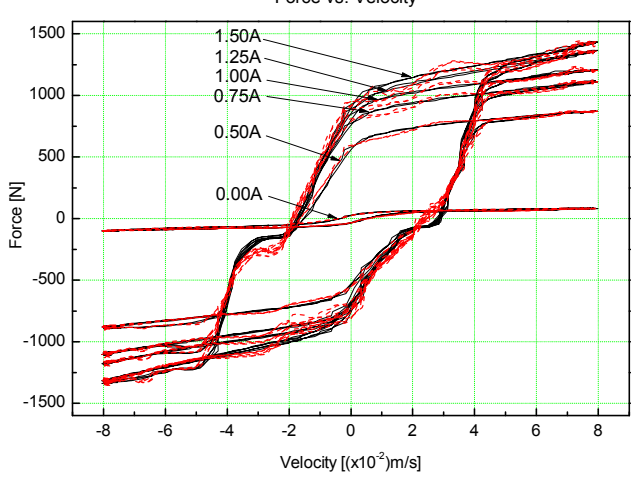

Figure 16. Comparison between the estimated force and actual damping force for an applied current range $(0,1.5) \mathrm{A}$ at a sinusoidal excitation (frequency $2.5 \mathrm{~Hz}$ and amplitude $0.005 \mathrm{~m}$ ).

Secondly, displacement excitations with a continuous variation of the frequency were generated to fully check the ability of the designed modeling method in case of varying excitation environments. In addition, experimental data were measured from the damping system, rig TR01, with the chirp displacement excitations of which the frequencies were varied from $1 \mathrm{~Hz}$ to $2.5 \mathrm{~Hz}$. Figures 17 and 18 depict the comparisons of the real damping responses and the estimated forces using the different models in cases: $0 \mathrm{~A}$ and $1.5 \mathrm{~A}$ of the applied current for the MR fluid damper coil. As seen in figures 17a and 18a, the damping behavior could not be modeled by using the Bingham model, Bouc-Wen model, or Hysteretic model. Here, the Bingham model could only predict the relation between damping force and time/ or displacement/or velocity as the one-to-one relation. Meanwhile, the Bouc-wen and Hysteretic models can only predict force for a particular damping system as the TR01 when their parameters are optimized with respect to this system. In contrast to the unfavorable modeling results in figures $17 \mathrm{a}$ and $18 \mathrm{a}$, figures $17 \mathrm{~b}$ and $18 \mathrm{~b}$ show a good damping force prediction using the designed BBM model. From these results, it is clearly that with the self-tuning ability, the BBM has enough strength to describe well the nonlinear behavior of the damper under various excitation environments, especially in case of low supplied current levels. 


\subsubsection{Investigation of self-sensing behavior of the TRO2 using the optimized BBM}

In this section, the ability of optimized BBM model is investigated when it is applied as a virtual force sensor to any damping system using the same MR fluid damper, such as the rig TR02, for the self-sensing behavior.

In the test rig TR02, the vibration was generated by the pneumatic cylinder and proportional valve of which the control signal was a voltage signal. This signal was a sinusoidal of which the frequency was in a range from 1 to $2.0 \mathrm{~Hz}$ while the amplitude was $3 \mathrm{~V}$ as:

$$
\text { ValveControlSignal }=A \sin (2 \pi f t) ; A=3 V ; f \in[1,2.0] \mathrm{Hz}
$$

There were two cases of working load: $3 \mathrm{~kg}$ and $9 \mathrm{~kg}$ while the supplied current for the MR fluid damper was changed from 0 to $2 \mathrm{~A}$ in order to create different test conditions. Consequently, setting parameters for experiments on the TR02 system are shown in Table 5.

\begin{tabular}{|c|c|c|c|c|}
\hline \multirow{2}{*}{ Test No. } & \multirow{2}{*}{$\begin{array}{c}\text { Working } \\
\text { load (kg) }\end{array}$} & Amplitude (V) & Frequency (Hz) & \multirow{2}{*}{$\begin{array}{c}\text { MR fluid } \\
\text { damper } \\
\text { current (A) }\end{array}$} \\
\cline { 3 - 4 } & & 3.0 & 1.0 & 0.0 \\
\hline Case 01 & 9 & 3.0 & 1.5 & 1.0 \\
\hline Case 02 & 3 & 3.0 & 2.0 & 2.0 \\
\hline Case 03 & 3 & &
\end{tabular}

Table 5. Test conditions on the TR02 for verification of the BBM with self-sensing behavior

A testing program for the BBM model verification using the rig TR02 was built in Simulink with the real-time toolbox of MATLAB as shown in Fig. 19. Experiments had been done on the TR02 in which the vibration was created as in Table 5 by using the program in Fig. 19. During the system operations, the real damping force caused by the vibration was measured by the load cell in order to make the comparison with the damping force 'measured' by the virtual force sensor - BBM. Consequently, the evaluation results of self-sensed force with respect to the test conditions are depicted on figures from $20 \mathrm{a}$ to $20 \mathrm{c}$.

As seen in these figures, the predicted performances using the BBM model were mostly close to the real damping performances. However, there were some predicting errors at the limits of damper compressions which can be seen in Fig. 20 as the tips of excitations. There are some reasons for these errors. The first reason is that the BBM model was optimized using experimental investigations on the rig TR01 of which the hardware included compliances and the control system included measuring noises. In addition, the rig TR02 is a damping system activated by using the pressurized pneumatic cylinder. Consequently, the changing of the generated excitations was fast, especially when the cylinder was extracted and then retracted which caused the damper compression limits. Therefore, there were some small errors in the estimated damping force near the damper compression limits.

From the results in Fig. 20, it is clear that the proposed self-sensing methodology based on the BBM model has strong ability to apply to damping control systems without using the force sensor. 

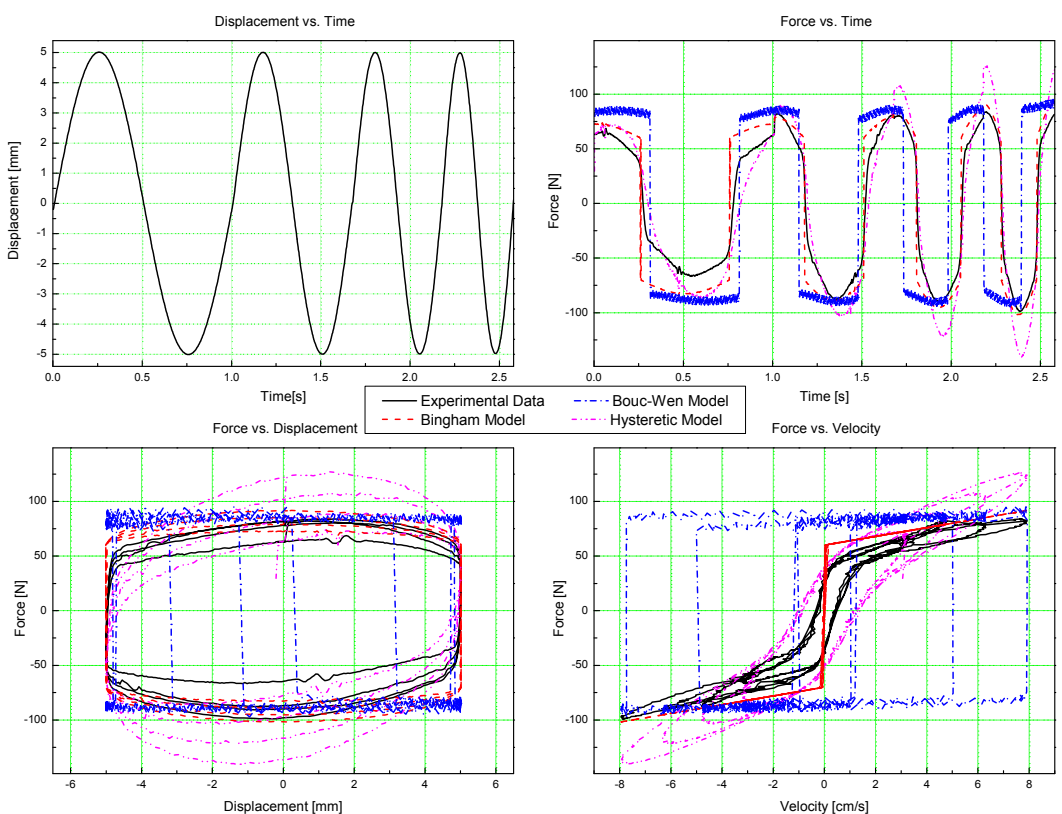

(a) Modeling results using the conventional models
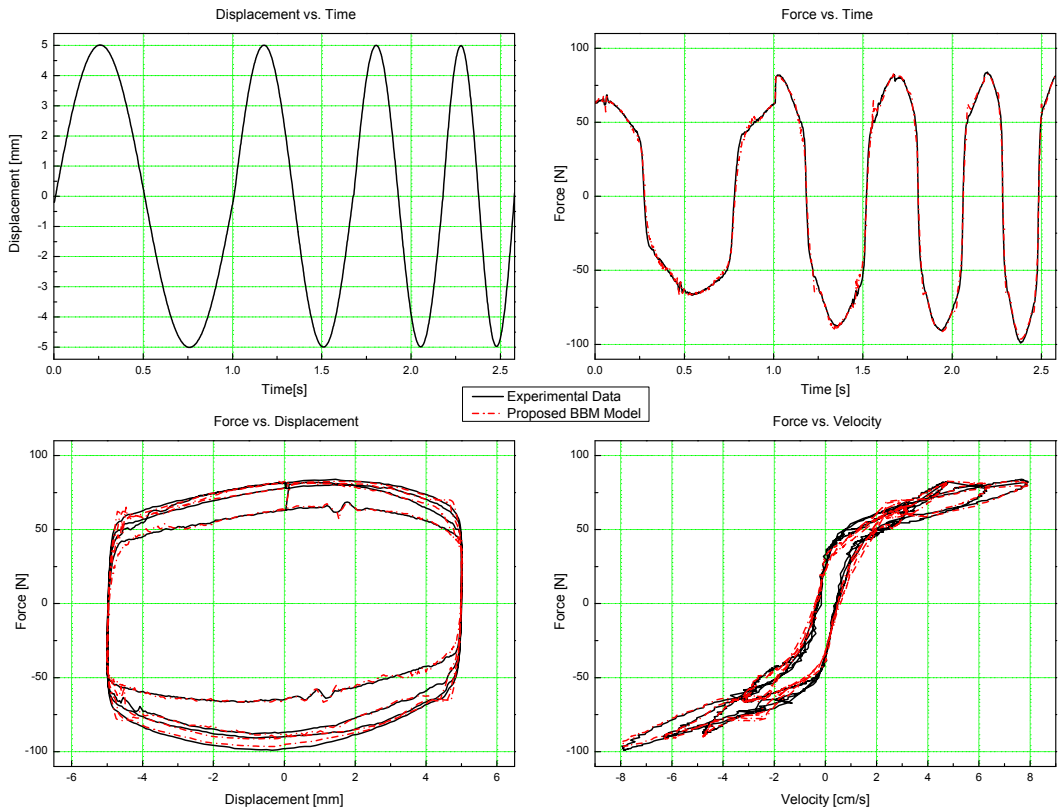

(b) Modeling results using the proposed BBM Model

Figure 17. Comparison between estimated forces and actual damping force for an applied current $0 \mathrm{~A}$ at a chirp excitation (frequency range $(1,2.5) \mathrm{Hz}$ and amplitude $5 \mathrm{~mm}$ ). 

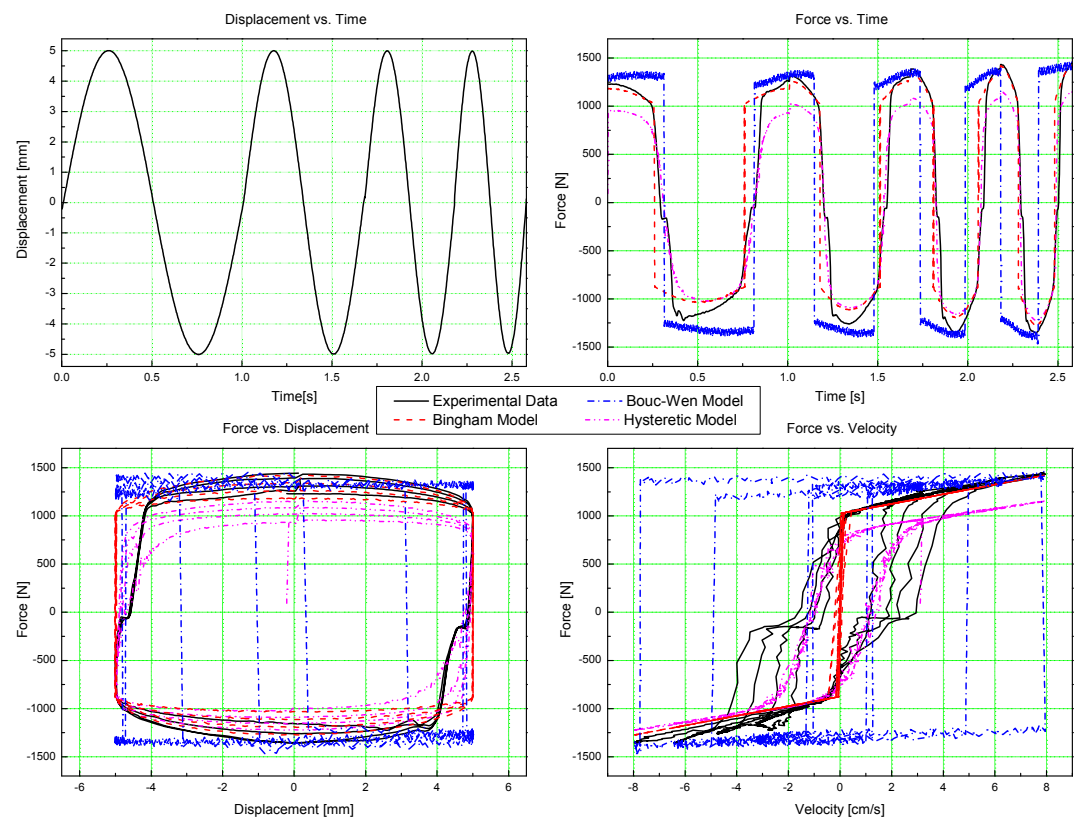

(a) Modeling results using the conventional models
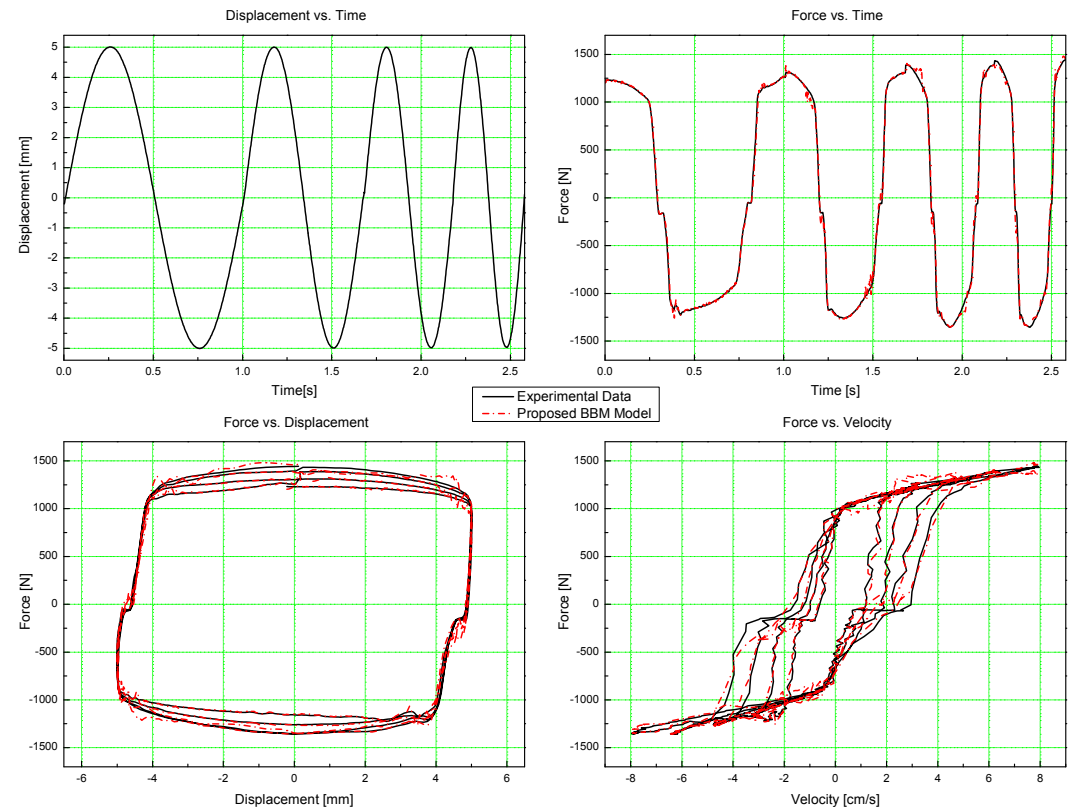

(b) Modeling results using the proposed BBM Model

Figure 18. Comparison between estimated forces and actual damping force for an applied current 1.5A at a chirp excitation (frequency range $(1,2.5) \mathrm{Hz}$ and amplitude $5 \mathrm{~mm}$ ). 


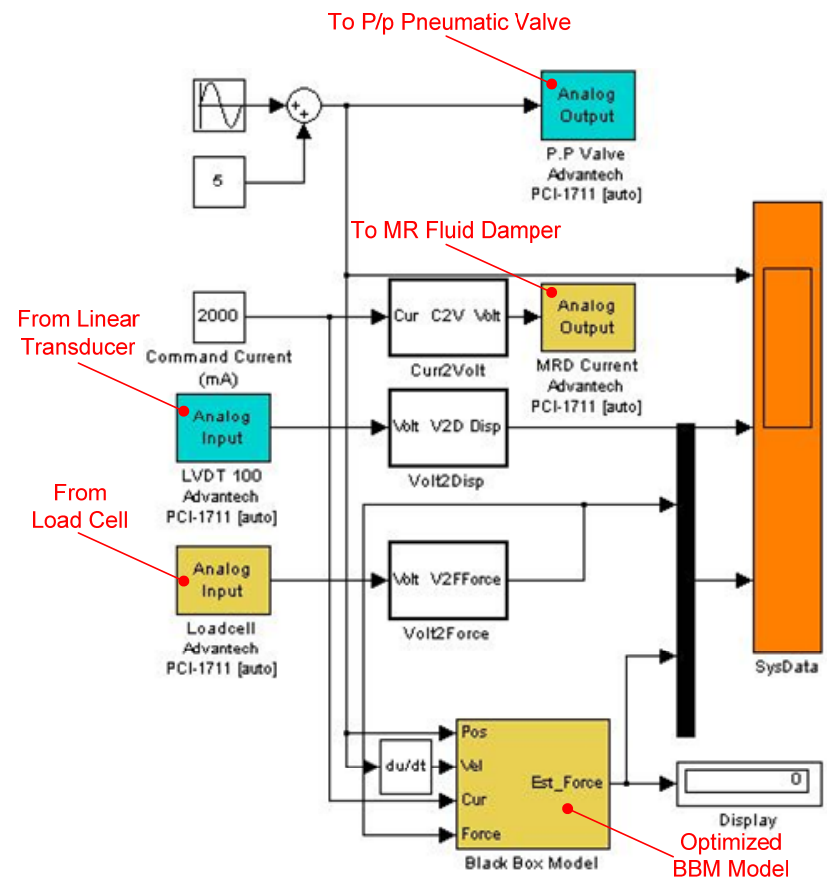

Figure 19. Simulink program for verification of the BBM sensor with self-sensing behavior.

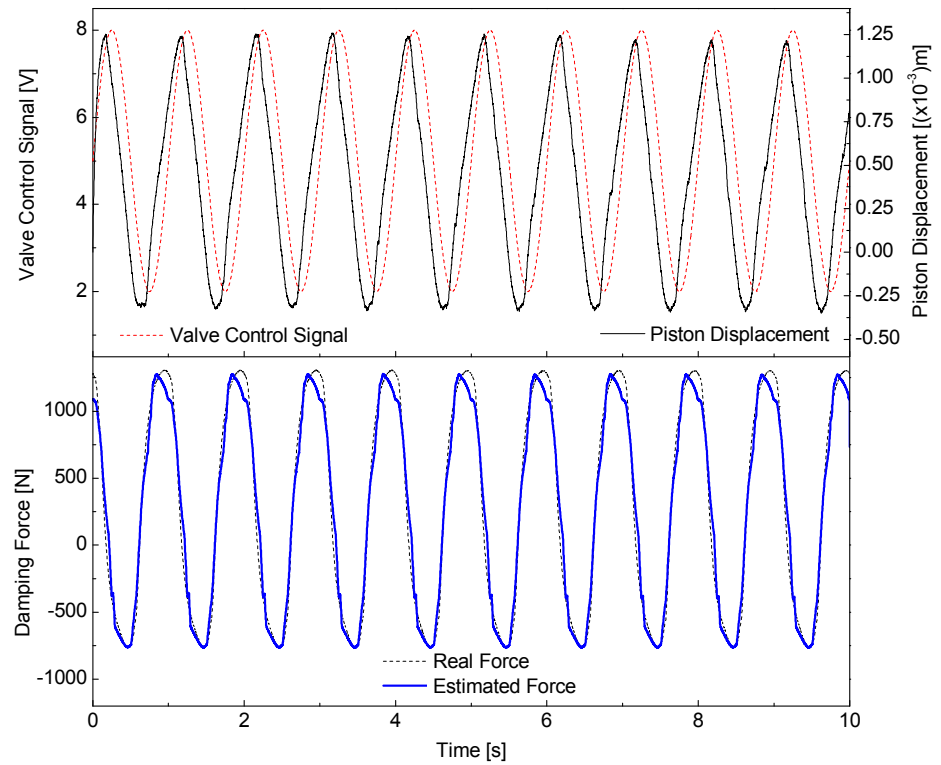

(a) Case 01 (Working load: 9kg; Vibrating control signal: (3V, 1Hz); Damper applied current: 0.0A) 


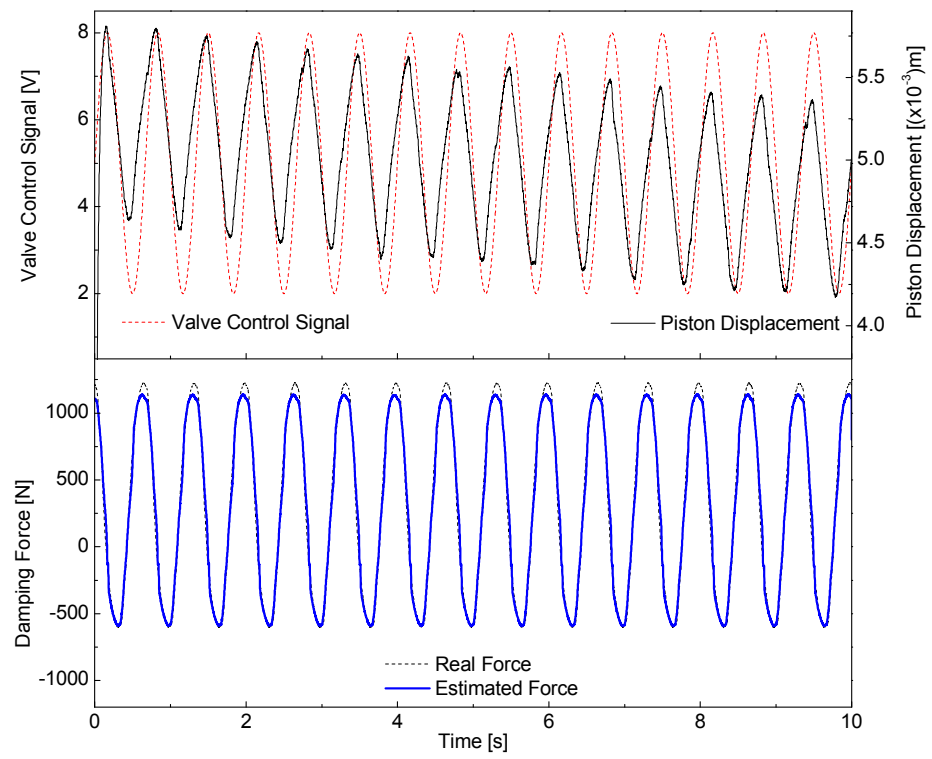

(b) Case 02 (Working load: 3kg; Vibrating control signal: (3V, 1.5Hz); Damper applied current: $1.0 \mathrm{~A}$ )

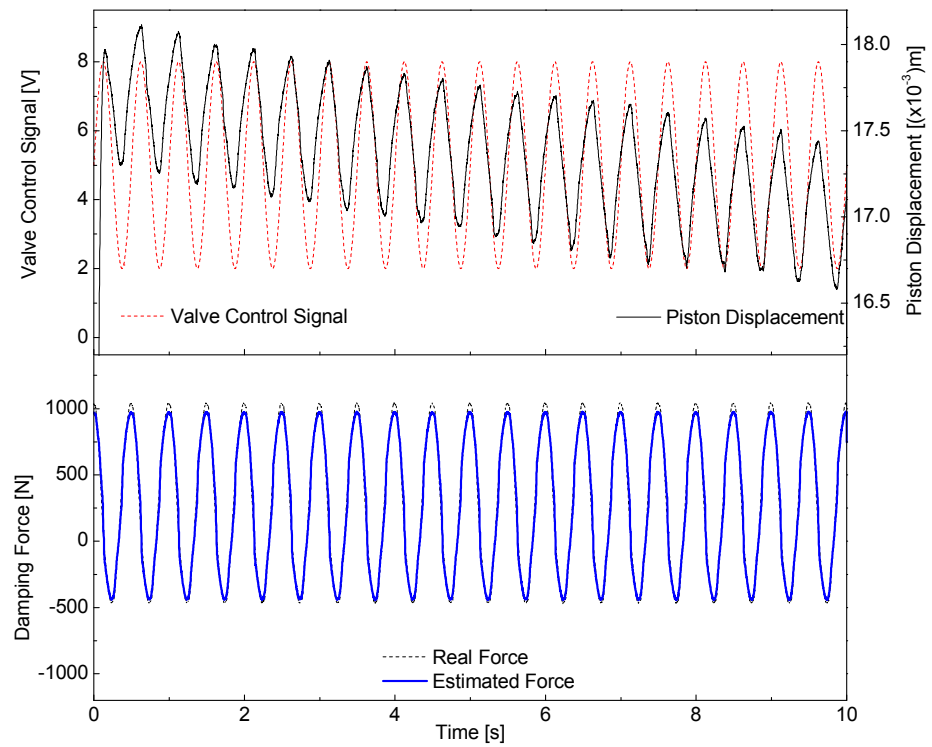

(c) Case 03 (Working load: 3kg; Vibrating control signal: (3V, 2.0Hz); Damper applied current: 2.0A)

Figure 20. Comparisons between the real and estimated damping forces of the TR02 system using the self-sensing method based on the optimized BBM. 


\section{Force sensorless damping control system design}

In this section, the force sensorless control technique, combined from the BBM and IBBM models, is developed to be applied to systems using this damper for damping control with force self-sensing behavior. The BBM model, which uses a self-tuning fuzzy system optimized by neural network technique, was designed to overcome the disadvantages of conventional models. The BBM built in the form of the simple fuzzy mapping laws is considered to estimate directly the damping force output with respect to the characteristics and inputs of the MR damper. In order to improve the accuracy of the suggested model, the back-propagation learning rule based on the gradient descent method has been used to train the fuzzy parameters to minimize a defined modeling error function. Next, the IBBM model is derived from the optimized BBM and suggested to use as an effective force controller. In addition, the IBBM structure is online adjusted with respect to the control error to improve the system performance.

Finally, the proposed controller based on the BBM and IBBM is built for the force sensorless damping system as shown in Fig. 21. Here, the BBM model works as a virtual force sensor while the IBBM takes part in direct damping control without using other control techniques.

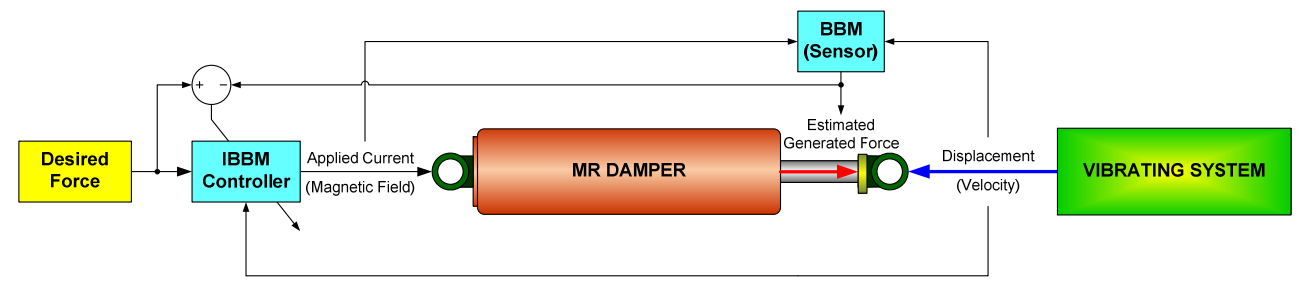

Figure 21. Structure of the proposed force sensorless damping control system.

\subsection{Design of IBBM model for the proposed force-sensorless damping control method}

Remark 3 (inverse model selection). Based on the advantages of the direct modeling method for the MR fluid damper using the black-box model, the inverse black-box model has been derived as the damping force controller. The BBM optimized by using neural network technique has been used to set initial parameters for the IBBM controller. The proposed inverse model estimates current levels should be applied to the MR fluid damper to create desired damping forces.

Remark 4 (force-sensorless control solution and model optimization). The proposed control method is designed for damping systems without using any force sensor. Hence, the optimized BBM is integrated in the control system as a virtual force sensor to estimate the generated damping force. This estimated force with the desired force are fed back to the IBBM controller to compute a control signal (current signal) to supply the MR fluid damper and, subsequently, to perform a closed-loop force-sensorless controller. In order to improve the control performance using the inverse model, the self-learning mechanism based on 
neural network technique is also integrated into the IBBM of which the parameters are continuously adjusted during the damping control process with respect to the control error minimization.

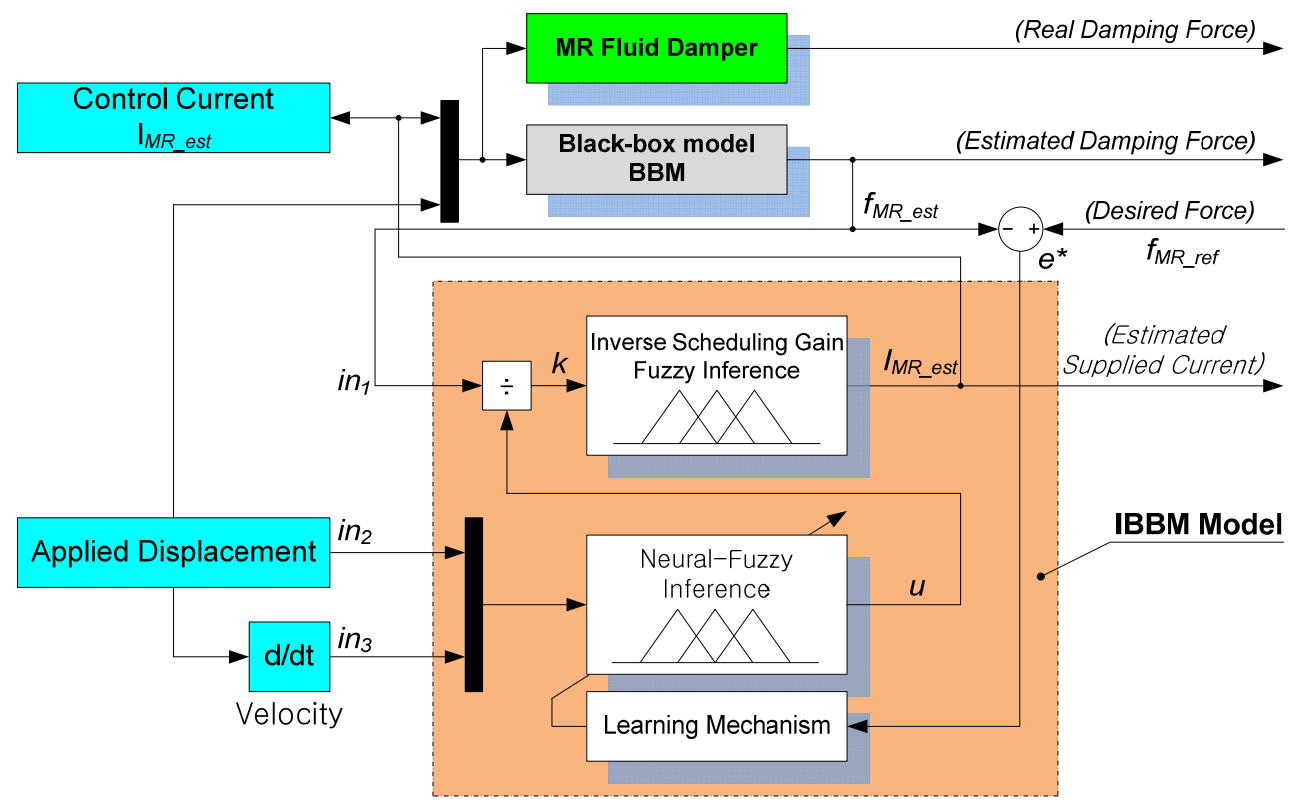

Figure 22. Structure of proposed force-sensorless damping control system based on BBM and IBBM.

Consequently, the force-sensorless control system is suggested as in Fig. 22. As seen in this figure, the proposed IBBM model contains two parts. The first part is a neural-fuzzy inference $\left(\mathrm{NFI}^{*}\right)$, which was derived from NFI system of the optimized BBM, to estimate the damping force $(u)$ caused by the damper rod displacement/velocity. The second part is an inverse scheduling gain fuzzy inference (ISGFI) which was developed from SGFI system of the optimized BBM. The ISGFI selects the current (IMR_est) level needed to supply for the MR fluid damper for obtaining the damping force level $(k)$. This damping force level can be computed from the damping force ( $\left.f_{M R_{-} e s t}\right)$ estimated by using the BBM and the estimated damping force $(u)$ caused by the damper rod displacement/velocity (see Fig. 22) as:

$$
k= \begin{cases}f_{M R_{-} e s t} / u & \text { if } u \neq 0 \\ 0 & \text { if } u=0\end{cases}
$$

For improving the IBBM control accuracy, an error function $\left(E^{*}\right)$ was derived from the difference between the damping force $\left(f_{M R_{-} \text {sst }}\right)$ measured by the BBM sensor and the desired force $\left(f_{M R_{-}} r e f\right)$. Therefore, the error function is defined as following equation:

$$
E^{*}=0.5\left(f_{M R_{-} e s t}-f_{M R_{-} r e f}\right)^{2}
$$




\subsubsection{Neural-Fuzzy inference $\left(N F I^{*}\right)$}

The NFI in the optimized BBM was used to construct the inverse model. Hence, the structure of the $\mathrm{NFI}^{*}$ in the IBBM is the same as the descriptions in section 3.2.1a except the initial MFs of the fuzzy inputs/output (Fig. 13). In the IBBM, these initial MFs of the NFI" are set as the optimized MFs of the NFI in the BBM which were shown in Fig. 15 of section 3.2.2.

Furthermore in the IBBM, the decisive factors of the NFI* input MFs, $a_{j}, b_{j}$, and the weights of $\mathrm{NFI}^{*}$ output, $w_{j}$, are automatically online-trained by using neural network technique during the damping force control process. Therefore, these affecting factors are updated as follows

$$
\left.\begin{array}{l}
\left.a_{j i}\right|_{t+1}=\left.a_{j i}\right|_{t}-\left.\eta_{a}^{*} \frac{\partial E^{*}}{\partial a_{j i}}\right|_{t} \\
\left.b_{j i}\right|_{t+1}=\left.b_{j i}\right|_{t}-\left.\eta_{b}^{*} \frac{\partial E^{*}}{\partial b_{j i}}\right|_{t} \\
\left.w_{k}\right|_{t+1}=\left.w_{k}\right|_{t}-\left.\eta_{w}^{*} \frac{\partial E^{*}}{\partial w_{k}}\right|_{t}
\end{array}\right\}
$$

where and are the learning rate which determine the speed of learning;

$E^{*}$ is the error function defined by (25).

The factor $\frac{\partial E^{*}}{\partial w_{k}}$ in equation (25) can be calculated as

$$
\frac{\partial E^{*}}{\partial w_{k}}=\frac{\partial E^{*}}{\partial f_{M R_{-} e s t}} \frac{\partial f_{M R_{-} e s t}}{\partial I_{M R_{-} e s t}} \frac{\partial I_{M R_{-} e s t}}{\partial k} \frac{\partial k}{\partial u} \frac{\partial u}{\partial w_{k}}
$$

where:

$$
\begin{aligned}
& \frac{\partial E^{*}}{\partial f_{M R_{-} e s t}}=e^{*}(t)=f_{\text {MR_est }}(t)-f_{M_{R_{-}} r e f}(t) \\
& \left.\frac{\partial f_{M R_{-} e s t}}{\partial I_{M R_{-} e s t}} \approx \frac{\delta f_{M R_{-} e s t}}{\delta I_{M R_{-} e s t}}\right|_{t} \\
& \left.\frac{\partial I_{M R \_e s t}}{\partial k} \approx \frac{\delta I_{M R_{\_} e s t}}{\delta k}\right|_{t} \\
& \frac{\partial k(t)}{\partial u(t)}=-\frac{f_{\text {MR_est }}}{u^{2}}
\end{aligned}
$$




$$
\frac{\partial u}{\partial w_{k}}=\frac{\mu\left(w_{k}\right)}{\left(\sum_{l=1}^{M} \mu\left(w_{l}\right)\right)}
$$

The next factors $\frac{\partial E^{*}}{\partial a_{j i}}$ in (26) can be computed by:

$$
\frac{\partial E^{*}}{\partial a_{j i}}=\frac{\partial E^{*}}{\partial u} \frac{\partial u}{\partial \mu_{j}\left(x_{i}\right)} \frac{\partial \mu_{j}\left(x_{i}\right)}{\partial a_{j i}}
$$

where:

$\frac{\partial E^{*}}{\partial u}$ is calculated by using equations from (28) to (31).

$$
\begin{gathered}
\frac{\partial u}{\partial \mu_{j}\left(x_{i}\right)}=\frac{\partial u}{\partial \mu\left(w_{k}\right)}=\frac{\sum_{l=1}^{M} \mu\left(w_{l}\right)\left(w_{k}-w_{l}\right)}{\left(\sum_{l=1}^{M} \mu\left(w_{l}\right)\right)^{2}} \\
\frac{\partial \mu_{j}\left(x_{i}\right)}{\partial a_{j i}}=\operatorname{sign}\left(x_{i}-a_{j i}\right) \frac{2}{b_{j i}}
\end{gathered}
$$

The final factor $\frac{\partial E^{*}}{\partial b_{j i}}$ in (26) can be found by:

$$
\frac{\partial E^{*}}{\partial b_{j i}}=\frac{\partial E^{*}}{\partial u} \frac{\partial u}{\partial \mu_{j}\left(x_{i}\right)} \frac{\partial \mu_{j}\left(x_{i}\right)}{\partial b_{j i}}
$$

where:

$\frac{\partial E^{*}}{\partial u}$ and $\frac{\partial u}{\partial \mu_{j}\left(x_{i}\right)}$ are calculated by using equations from (28) to (31), and (34), respectively.

$$
\frac{\partial \mu_{j}\left(x_{i}\right)}{\partial b_{j i}}=\frac{2\left|x_{i}-a_{j i}\right|}{b_{j i}^{2}}
$$

By using the above self-learning algorithm (from (26) to (37)), the NFI* can work more precisely in estimating the damping force $(u)$ with respect to the applied displacement/velocity. 


\subsubsection{Inverse scheduling gain fuzzy inference (ISGFI)}

The ISGFI system, designed with a single input $(k)$ and a single output (IMR_est), works as an intelligent switch to select the current (IMR_est) level needed to supply for the MR fluid damper with respect to the damping force level $(k)$. This fuzzy system was obtained from the SGFI mechanism of the BBM design (section 3.2.1) in which the SGFI input/output became the ISGFI output/input. Consequently, the input/output MFs and rule table of the ISGFI system were set as in Fig. 23 and Table 6, respectively.

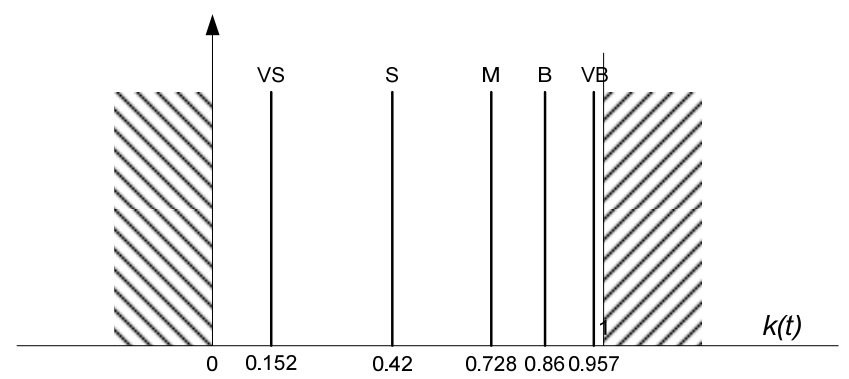

(a) ISGFI input: $k(t)$

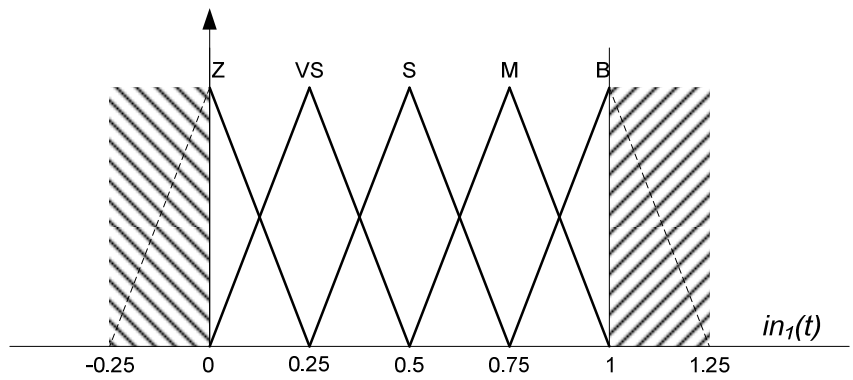

(b) ISGFI output: IMR_est $(t)$

Figure 23. MFs of the ISGFI inputs and output.

\begin{tabular}{|c|c|c|c|c|c|}
\hline Damping Force Gain $(k)$ & VS & S & M & B & VB \\
\hline Estimated Supplied Current (IMr_est $)$ & Z & VS & S & M & B \\
\hline
\end{tabular}

Table 6. Rules table for the inverse scheduling gain inference of the inverse black box model

\subsection{Verifications}

\subsubsection{Checking the control ability of a damping system using the designed IBBM}

As the descriptions for the proposed force-sensorless control method, the designed IBBM controller was firstly examined by simulations before its application to the real-time damping control. Therefore, a simulating scheme for the IBBM controller validation was built in Simulink/MATLAB as in Fig. 24. As seen in this figure, the validating damping 
control system contains three main blocks. The two blocks labeled as 'BBM1' and 'BBM2' are similar and represent for the optimized BBM model which was obtained in section 3.2. These blocks then function as the actual MR fluid dampers. The remained block is the IBBM controller which was designed from section 4.1.

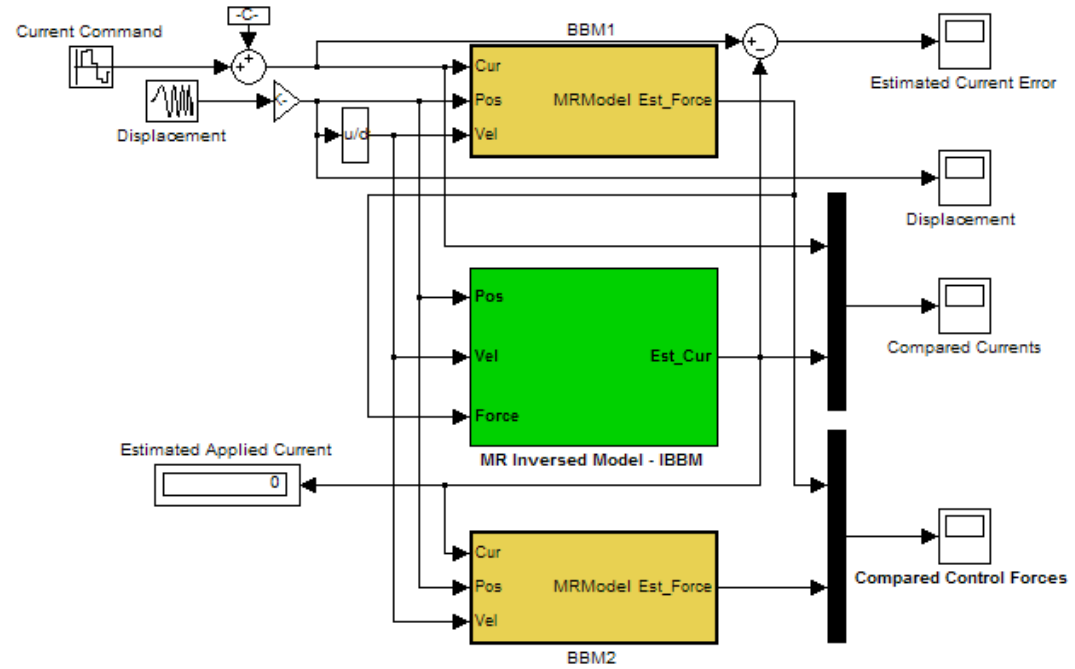

Figure 24. Simulation program for testing the IBBM controller.

The goal of the validation process is using the IBBM controller to control the second damper model, BBM2, to create the damping force to follow the reference force given from the first damper model, BBM1. Therefore, a displacement/velocity signal was generated and input into both the two damper models and the force controller. A current command signal was applied to the first damper model, BBM1. The output of this model, called the first simulated damping force, was used as a reference force signal for the damping system based on the second damper model, BBM2, and the IBBM controller. Hence, corresponding to a force command sent from the BBM1, the IBBM generated a simulated current command to control the damper model BBM2. This simulated current was then fed into the BBM2 together with the applied displacement/velocity to produce the second simulated damping force. As a result, the validation process carried out the comparison between the reference current command and simulated current command obtained from the IBBM, and the comparison between the first and second simulated damping forces.

The simulation results are displayed in Fig. 25. The results show that almost not only the simulated current command coincided with the reference current command, but also the simulated damping force of the BBM2 coincided with the simulated damping force obtained from the BBM1. As the result, it points out that the damping system using the self-learning IBBM controller with the optimized BBM virtual sensor can control accurately the MR fluid damper for a good tracking force performance. In the next section, experiments on the test rig TR02 have been carried out in order to verify the real-time control ability of the proposed force-sensorless damping control method. 

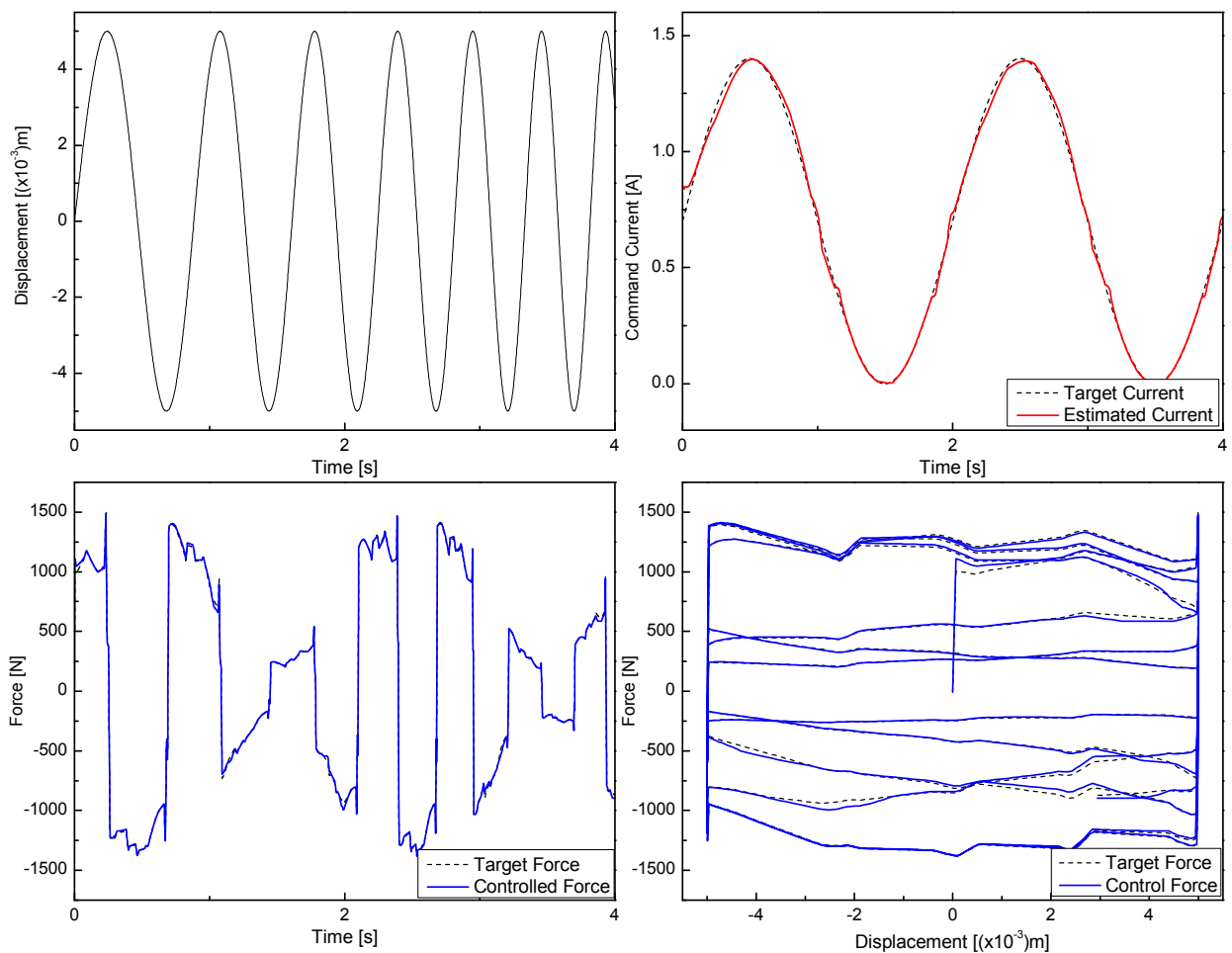

Figure 25. Comparison of simulation results between estimated current command with given current command corresponding to the damping force output.

\subsubsection{Verification of the force-sensorless control system based on the BBM and IBBM}

Based on the advanced characteristics of the designed BBM and IBBM models which were proven to be effective in the previous sections, the proposed force-sensorless control methodology was applied to the system TR02 for the real-time damping force control. Here, a harmonic excitation was applied to the damping system through the pneumatic cylinder controlled by the proportional valve. Meanwhile, a desired force performance was given in order to validate the damping control ability. Therefore, the control signal (voltage) for the pneumatic valve and the reference damping force were given as equations (38) and (39), respectively.

$$
\begin{gathered}
\text { ValveControlSignal }=5 \sin (\pi t+\pi / 2)(V) \\
\text { ReferenceForce }=A \sin (\pi t)(N)
\end{gathered}
$$

here: the amplitude $A$ of the reference force signal was set at two values: $500 \mathrm{~N}$ and $1000 \mathrm{~N}$ to perform two testing cases. The working load condition was set with a $9 \mathrm{~kg}$ of load. 
The control program for the damping system TR02 was built in Simulink with the real-time toolbox of MATLAB as shown in Fig. 26. The program contained two main blocks in which the first one is the motion generating block to generate the system displacement while the second one is the damping control system to ensure that the system could track the desired damping force by only using the IBBM controller and BBM sensor, without using the mechanical force sensor (load cell). In this control system, the optimized BBM estimated the damping force and fed back to the self-learning IBBM controller to create the current control signal for the MR fluid damper. In order to evaluate the control performance, the load cell was used separately with the controller to measure the actual damping force.

Experiments on the TR02 controlled by the system in Fig. 26 had been done under the testing conditions as shown in (38) and (39). Consequently, the validating results for the force-sensorless damping control system based on the BBM and IBBM corresponding to the two testing cases, $500 \mathrm{~N}$ and $1000 \mathrm{~N}$ of the desire force amplitude, are shown in figures $27 \mathrm{a}$ and $27 \mathrm{~b}$, respectively. The figures show that the system using the IBBM controller with the virtual sensor BBM tracked the desired damping force well. From the results, it strongly indicates that the damping force can be completely controllable by this proposed control methodology.

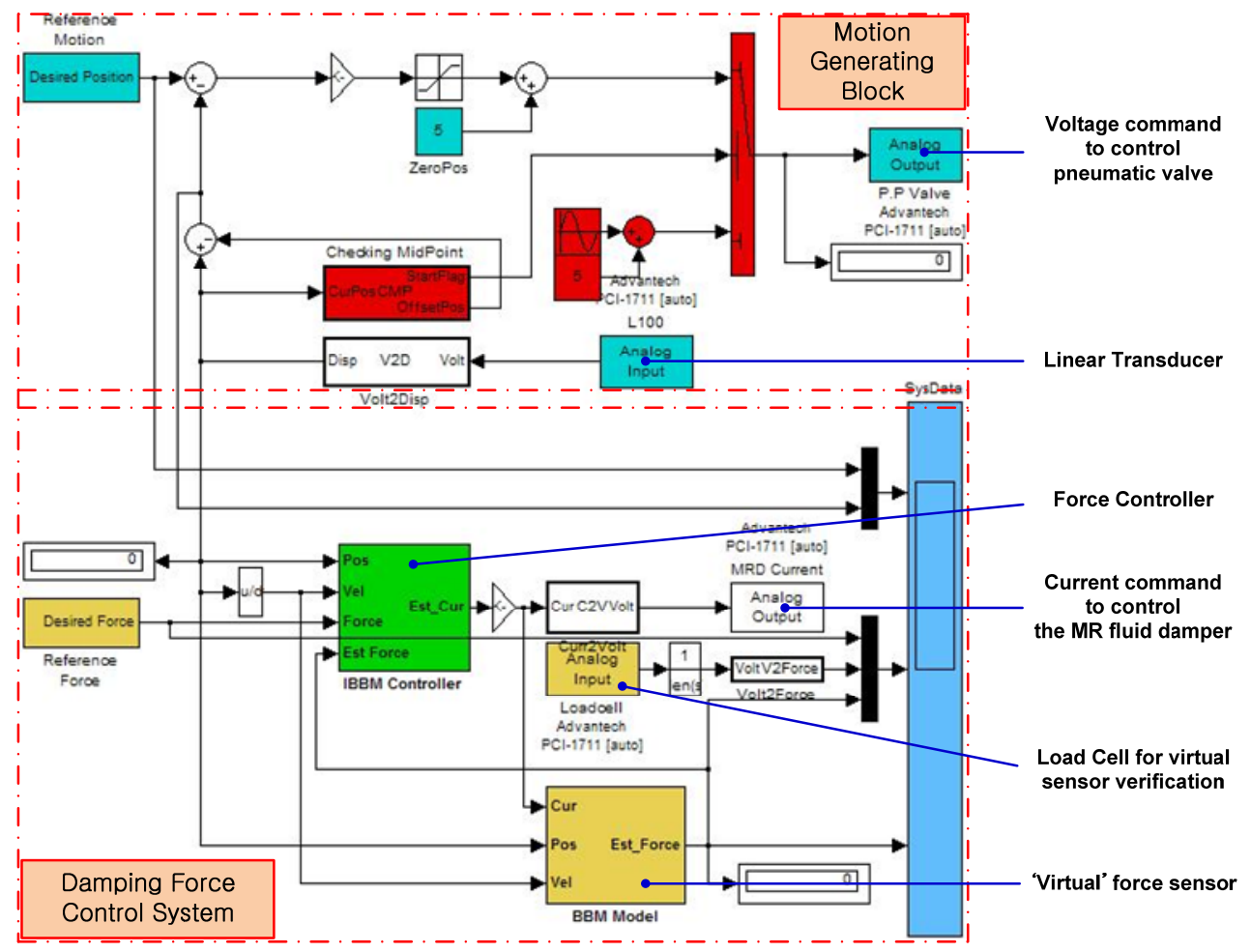

Figure 26. Simulink program for damping force control based on IBBM controller and BBM sensor. 


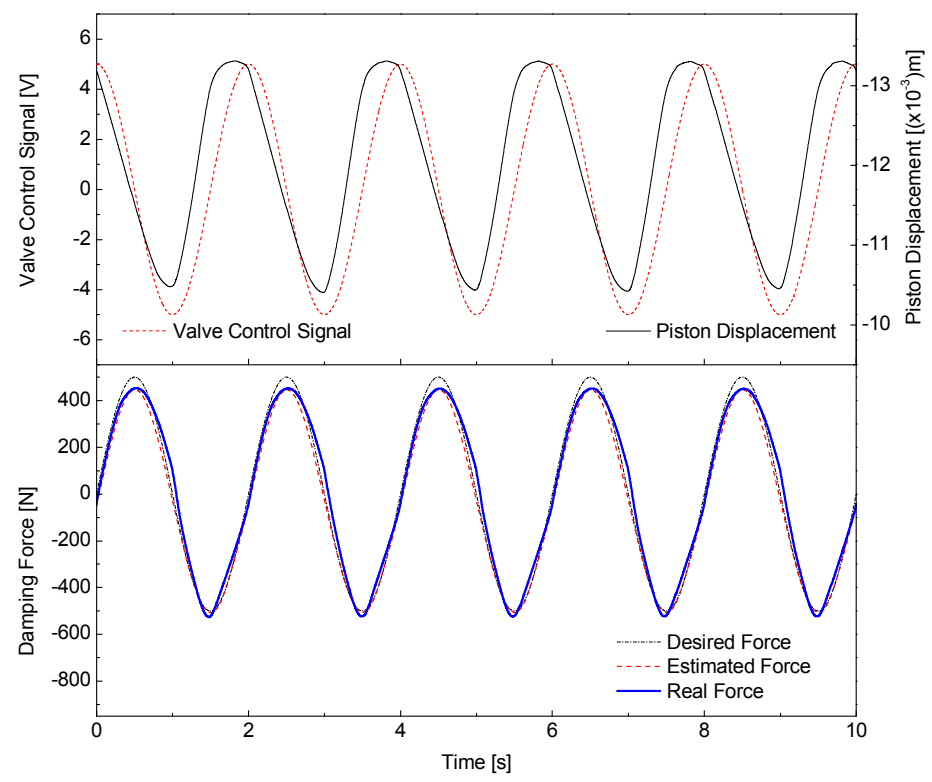

(a) Case 1 (Working load: 9kg; Vibrating control signal: $\left(5 \mathrm{~V}, 0.5 \mathrm{~Hz}, 90^{\circ}\right)$; Reference damping force: $\left.\left(500 \mathrm{~N}, 0.5 \mathrm{~Hz}, 0^{\circ}\right)\right)$

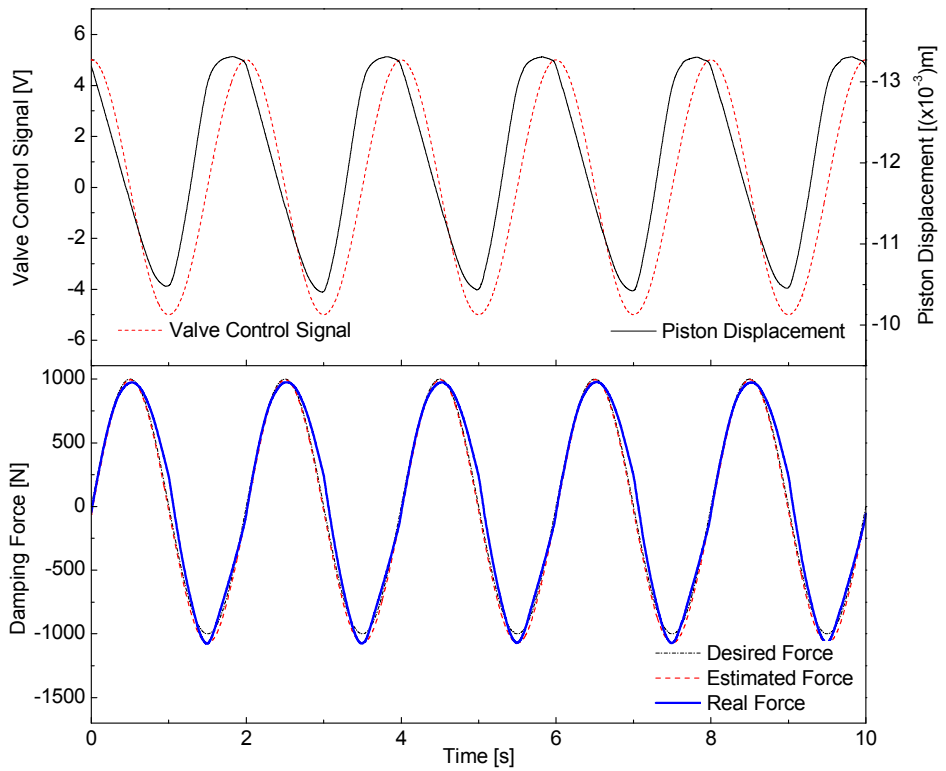

(b) Case 2 (Working load: 9kg; Vibrating control signal: $\left(5 \mathrm{~V}, 0.5 \mathrm{~Hz}, 90^{\circ}\right)$; Reference damping force: $\left.\left(1000 \mathrm{~N}, 0.5 \mathrm{~Hz}, 0^{\circ}\right)\right)$

Figure 27. Damping force control performance using IBBM controller and BBM sensor. 


\section{Conclusions}

In this chapter, physical phenomena of a MR fluid damper have been carefully investigated through both experimental data and modeling methodologies. Furthermore, a novel forcesensorless control method was proposed and successfully applied to the damping system using the MR fluid damper and without using any mechanical force sensor. The designed force control system is based on the optimized BBM model, functioning as the virtual force sensor, and the IBBM model, functioning as the self-learning force controller.

The two test rigs using the MR fluid damper have been fabricated in order to design the models as well as to evaluate the proposed controller. The BBM model is built as the simple direct modeling method and optimized by using neural network technique. The IBBM controller is the direct inverse model of the optimized BBM. In addition, the IBBM has the online self-learning ability by using neural network, consequently, improving the system performance. As the result, the optimized BBM and IBBM models have a strong ability to be applied to damping systems using the MR fluid damper as a virtual force sensor and a simple direct controller, respectively.

The modeling as well as control validation process has been done by both the simulations and real-time experiments. Based on the simulation and experimental results, it is clear that the optimized BBM can predict the force-displacement behavior of the MR damper with high precision while the IBBM can control the damping system to follow the desired damping force well. As a result, the proposed force-sensorless damping control methodology can become an effective and economical solution for practical MR fluid damper applications in the near future.

\section{Author details}

D. Q. Truong and K. K. Ahn*

School of Mechanical Engineering, University of Ulsan, Korea

\section{References}

[1] Karnopp D, Crosby M.J, Farwood R.A (1974) Vibration control using semi-active force generators. ASME j. eng. ind. 96(2): 619-626.

[2] Yi K, Song B.S (1999) A new adaptive sky-hook control of vehicle semi-active suspensions. Proc. of IMechE, Part D: j. auto. eng. 213(3): 293-303.

[3] Kawashima K, Unjoh S, Shimizu K (1992) Experiments on Dynamics Characteristics of Variable Damper. Proc. of the japan national symp. on structural response control, Tokyo, Japan: 121.

[4] Mizuno T, Kobori T, Hirai J, Matsunaga Y, Niwa N (1992) Development of Adjustable Hydraulic Dampers for Seismic Response Control of Large Structure. ASME PVP conf.: 163-170.

\footnotetext{
* Corresponding Author
} 
[5] Gavin H.P, Hose Y.D, Hanson R.D (1994) Design and Control of Electrorheological Dampers. Proc. of the first world conf. on structural control, Pasadena, CA: 83-92.

[6] Park W.C, Choi S.B, Suh M.S (1999) Material characteristics of an ER fluid and its influence on dampingforces of an ER damper. Part I: material characteristics. Materials \& design 20(6): 317-323.

[7] Park W.C, Choi S.B, Suh M.S (1999) Material characteristics of an ER fluid and its influence on dampingforces of an ER damper. Part II: damping forces. Materials \& design 20(6): 325-330.

[8] Choi S.B, Choi Y.T (1948) Sliding Model Control of a Shear-Mode Type ER Engine Mount. KSME Int. j. 13(1): 26-33.

[9] Rabinow J (1948) Proceedings of the AIEE trans. 67: 1308-1315.

[10] Rabinow J (1951) US Patent 2,575,360.

[11] Carlson J.D, Chrzan M.J (1994) Magnetorheological Fluid Dampers. U.S. Patent 5277281.

[12] Carlson J.D, Weiss K.D (1994) A growing attraction to magnetic fluids. J. machine design 66(15): 61-64.

[13] S.J Dyke, Spencer B.F. Jr, Sain M.K, Carlson J.D (1996) Modelling and control of magneto-rheological fluid dampers for seismic response reduction. Smart material and structures 5: 565-575.

[14] Boelter R., Janocha H (1998) Performance of long-stroke and low-stroke MR fluid damper. Proc. of SPIE, smart structures and materials: passive damping and isolation, San Diego, CA: 303-313.

[15] Carlson J.D, Jolly M.R (2000) MR fluid, foam and elastomer devices. Mechatronics 10: 555-569.

[16] Hong S.R, Choi S.B, Choi Y.T, Wereley N.M (2005) Non-dimensional analysis and design of a magnetorheological damper. J. of sound vib. 288(4): 847-863.

[17] Choi K.M, Jung H.J, Cho S.W, Lee I.W (2007) Application of smart passive damping system using MR damper to highway bridge structure. KSME Int. j. 21(6): 870-874.

[18] Spelta C, Previdi F, Savaresi S.M, Fraternale G, Gaudiano N (2009) Control of magnetorheological dampers for vibration reduction in a washing machine. Mechatronics 19(3): 410-421.

[19] Savaresi S.M, Bittanti S, Montiglio M (2005) Identification of semi-physical and blackbox non-linear models: the case of MR-dampers for vehicles control. Automatica 41(1): 113-127.

[20] Stanway R, Sproston J.L, Stevens N.G (1987) Non-linear Modelling of an Electrorheological Vibration Damper. J. of electrostatics 20(2): 167-184.

[21] Spencer B.F, Dyke S.J, Sain M.K, Carlson J.D (1996) Phenomenological Model of a Magneto-Rheological Damper. ASCE J. of eng. mech. 123(3): 230-238.

[22] Choi S.B, Lee S.K (2001) A Hysteresis Model for the Field- dependent Damping Force of a Magneto-rheological Damper. J. of sound vib. 245(2): 375-383.

[23] Dominguez A, Sedaghati R, Stiharu I (2004) Modelling the hysteresis phenomenon of magnetorheological dampers. Smart mat. struc. 13(6): 1351-1361. 
[24] Kwok N.M, Ha Q.P, Nguyen T.H, Li J, Samali B (2006) A Novel Hysteretic Model for Magneto-Rheological Fluid Damper and Parameter Identification Using Particle Swarm Optimization. Sens. actuators A 132(2): 441-451.

[25] Kwok N.M, Ha Q.P, Nguyen M.T, Li J, Samali B (2007) Bouc-Wen Model Parameter Identification for a MR Fluid Damper Using Computationally Efficient GA. ISA Trans. 46(2): 167-179.

[26] Chang C.C, Roschke P (1998) Neural network modeling of a magnetorheological damper. J. intell. mater. syst. struct. 9(9): 755-764.

[27] Schurter K.C, Roschke P.N (2000) Fuzzy Modeling of A Magneto-Rheological Damper Using Anfis. Proc. of IEEE int. conf. on fuzzy sys.: 122-127.

[28] Wang D.H, Liao W.H (2001) Neural Network Modeling and Controllers for Magnetorheological Fluid Dampers. Proc. of 10th IEEE int. conf. on fuzzy sys.: 1323-1326.

[29] Wang D.H, Liao W.H (2005) Modeling and Control of Magneto-Rheological Fluid Damper Using Neural Networks. Smart mat. struc. 14(1): 111-126.

[30] Hanson B, Levesley M (2004) Self-sensing applications for electromagnetic actuators. Sens. actuators A. 116(2): 345-351.

[31] Mizuno T, Ishii T, Araki K (1998) Self-sensing magnetic suspension using hysteresis amplifiers. Con. eng. prac. 6(9): 1133-1140.

[32] Lama K.H, Chena Z.H, Nia Y.Q, Chanb H.L.W (2010) A magnetorheological damper capable of force and displacement sensing. Sens. actuators A. 158(1): 51-59.

[33] Ahn K.K, Truong D.Q, Thanh T.Q, Lee B.R (2008) Online Self Tuning Fuzzy PID Control for Hydraulic Load Simulator. Proc. of the IMechE - part I: j. sys. and con. eng. 222(2): 81-95.

[34] Truong D.Q, Ahn K.K (2011) Parallel Control for Electro-Hydraulic Load Simulator Using Online Self Tuning Fuzzy PID Technique. Asian j. con. 13(4): 522-541.

[35] Ahn K.K, Truong D.Q, Islam M.A (2009) Modeling of a Magneto-Rheological (MR) Fluid Damper using a Self Tuning Fuzzy Mechanism. J. of mech. sci. and tech. 23(5): 1485-1499.

[36] Truong D.Q, Ahn K.K (2010) Identification and application of black-box model for a self-sensing damping system using a magneto-rheological fluid damper. Sens. actuators A. 161(1-2): 305-321.

[37] Shames I.H, Cozzarelli F.A (1992) Elastic and Inelastic Stress Analysis. Prentice Hall, Englewood Cliffs, New Jersey 120-122 p.

[38] Gamota D.R, Filisko F.E (1991) Dynamic Mechanical Studies of Electrorheological Materials: Moderate Frequencies. J. of rheology 35(3): 399-425. 